\title{
Two-Step Numerical Simulation of the Heat Transfer from a Flat Plate to a Swirling Jet Flow from a Rotating Pipe
}

August 24, 2019

\begin{abstract}
Purpose: Impinging jets have been widely studied and the addition of swirl has been found to be beneficial to heat transfer. Since there is no literature on RANS nor experimental data of swirling jet flows generated by a rotating pipe, this paper attempts to fill such gap by providing results on the performance of this type of design.

Design/methodology/approach: Since the flow has a different behaviour at different parts of the design, the same turbulent model cannot be used for the full domain. To overcome this complexity, the simulation is split into two coupled stages. This is an alternative to use the costly Reynold Stress Model (RSM) for the rotating pipe simulation and the SST $\mathrm{k}-\omega$ model for the impingement.

Findings: To induce swirl by rotating pipes with swirl intensity ranging from 0 to 0.5 affects the velocity profiles but not noticeably the spreading angle. The heat transfer is increased with respect to a non-swirling flow only at short nozzle-to-plate distances $H / D<6$, where $H$ is the distance and $D$ is the diameter of the pipe. For the impinging zone, the highest average heat transfer is achieved at $H / D=5$ with swirl intensity $S=0.5$. This is the highest swirl studied in this work.

Research limitations/implications: High-fidelity simulations or experimental analysis may provide reliable data for higher swirl intensities, which is not covered in this work with RANS.

Practical implications: This two-step approach and the data provided is of interest to other related investigations (e.g. using arrays of jets or other surfaces than flat plates).

Originality/value: This paper is the first of its kind RANS simulation of the heat transfer from a flat plate to a swirling impinging jet flow issuing from a rotating pipe. An extensive study of these CFD simulations has been carried out with the emphasis of splitting the large domain into two parts to facilitate the use of different turbulent models and periodic boundary conditions for the flow confined in the pipe.
\end{abstract}

Keywords: Heat transfer; Numerical simulations; Impinging jets; Turbulent flows; Heated plates; Swirling jets; CFD

Journal: International Journal of Numerical Methods for Heat and Fluid Flow. Submitted to the special issue Fluid Flow, Heat and Mass Transfer through Passages with Complex Geometries for Advanced Technological Applications.

Corresponding author(s): - 


\section{Introduction and Motivation}

Swirling flows in pipes can be found in many industrial applications, such as in erosion damage reduction [1], chemical engineering [2] or dehydration systems in multi-phase flows [3]. Another application of this type of flow is heat transfer by impingement. Impinging jets have been extensively studied for cooling in electronic components [4, 5], surface varied plates [6] or impinging gases and flame jets $[7,8]$, amongst many others. The improvement of the existing impinging jet mechanisms has also been studied extensively. However, to the knowledge of the authors, there is no work regarding the effect of using rotating pipes for swirling flow generation as impinging jet flows, and this has motivated the present study. The only reference found in the literature is a preliminary analysis on the impact of rotating pipe boundary conditions in LES simulations of impinging jet flows in [9]. The results do not match accurately experimental data in the validation of their heat transfer to a non-swirling jet, particularly struggling to simulate the secondary peak in the Nusselt distribution at short nozzle-to-plate distance. This has been actually identified as a challenging problem in CFD by other authors [10]. The additional objective of the present work is to discuss the drawbacks and advantages in comparison with other swirl flow generators: the use of pipes with spirals or vane-type swirl generators [11], swirl strips in pipes [12], angled blades to force rotation [13,14] and the use of tangential jets to impart the swirl [15]. For this purpose, a novel computational methodology is suggested by means of splitting the large simulation into two stages, referred to as Simulation 1 and Simulation 2, as shown in Figure 1. Despite this approach is novel in the computation of impinging jets, the use of other simulations or data to impose boundary conditions can be found in other fields such as combustion science. For instance, in [16], the combustion in a motored single-cylinder engine is simulated, using the full simulated system to generate boundary conditions, in order to apply more realistic profiles and thermodynamic conditions to inlet data. Similarly experimental data [17] is used as part of the boundary conditions for the simulation of a single-cylinder light-duty diesel engine. In [18], inflow boundary conditions for a Diesel Spray simulations (valid either in RANS or LES) are modelled, in order to alleviate the drawbacks in the 3D modelling of these sprays near the nozzle exit. Another interesting application is [19], where RANS and LES are coupled to achieve a good balance of predictive capabilities and computational costs in the CFD simulation of the flow in the compressor/combustor interface of a gas turbine engine.

It is found that a simple mechanism such as a rotating pipe is not recorded in the literature, except other ways of inducing swirl in flows to improve the heat transfer [12, 20, 11, 13, 6, 21]. Amongst those options as described in the literature, a pipe with a forced angular velocity seems straight forward in manufacturing and is an affordable piece of laboratory equipment without machining or assembly of extra complex components. Also, there is a great range of swirl intensity available, since one only needs to increase the angular velocity of the pipe by means of a step-wise motor. With other mechanisms, such as the oriented blades or angled spirals strips, there is a maximum angle that cannot be surpassed. On the other hand, the use of a rotating pipe long enough to guarantee a fully-developed flow can be a limitation to some applications, as well as the requirement of additional power consumption. Summarising, the suggested mechanism can be a flexible method, and of interest if the specific limitations are acceptable for the application. This supports the interest in the present computational study. 


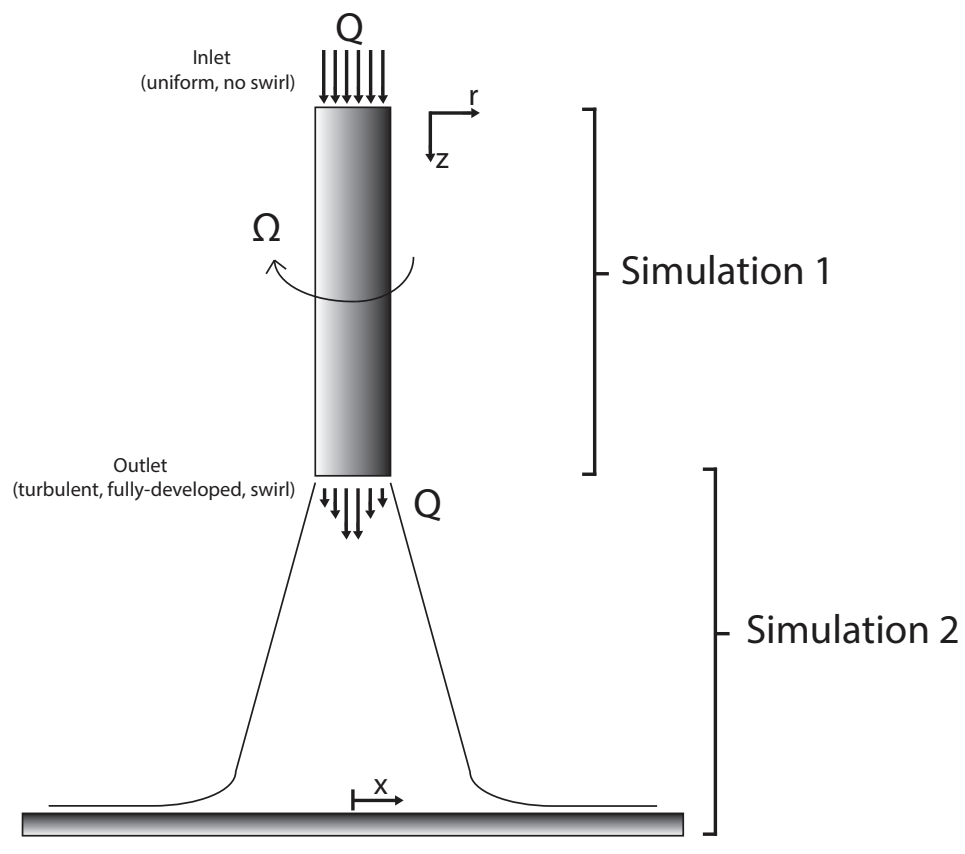

Figure 1: Sketch of Simulation 182 . Note that the $x$ and $r$ axis are the same in practice and with origin in the axisymmetry axis, but for the sake of avoiding confusion in the following plots and data tables, $r$ is used in Simulation 1 and $x$ for Simulation 2.

The number of experimental studies on swirling jet flows greatly surpass the computational ones, with a variety of swirl injection methods. There are significant work on the experimental analysis of confined swirling flows. One of the first attempts on swirling flows appears in [22], showing experimentally that the flow suffers some stabilisation when the a round pipe is spinning on its axis. In [23], a long pipe rotating about its axis too was analysed by measurements and observations of the flow pattern, losses within the pipe, velocity and pressure distributions across different pipe sections. In [24], the velocity and turbulence was the objective of the investigation in rotating pipes, using the hot-wire technique to measure velocity and Reynolds stress components. Further continuation of research in swirling flows confined in rotating pipes can be found in [25] forming the reference data for the validation of computational results in the present paper. In [25], an experimental study with Laser Doppler Velocimetry (LDV) measurements was been carried out in order to measure mean velocity profiles, the components of the Reynolds stress, and velocity fluctuations. Such reference hence provides a very complete analysis on the properties of swirling flows.

There is a vast amount of literature concerning computational simulations of swirling flows [26, 27, 28, 29, 30, 31] and the turbulence models used for confined swirling flows when the RANS equations are employed. In general, the Reynolds Stress Model (RSM) gives the best performance in these works. However, in [28] a modified $k-\epsilon$ turbulence model is used for a swirling flow in a straight pipe, based on the improvement of the existing $k-\epsilon$ by analysing the flow from an anisotropic viewpoint modifying the closure equations. Other authors also tried modifications in the model closure equations by adding curvature corrections [32]. In [33], an exhaustive analysis on the aspects of each turbulence closure is carried out to understand the generation of the axial and 
azimuthal velocity profiles. It also pointed out that traditional two-equation turbulence models are not capable to deal with the effect of rotation in axial and azimuthal velocities. Two-dimensional explicit algebraic stress models, which only have a quadratic tensorial non-linearity, are able to simulate accurately the axial velocity profile, but fail to mimic the azimuthal one, and cubic nonlinearities are needed to overcome the issue. It is claimed in such paper that the reason might be related to the underlying effect from conventional algebraic models and wall treatments, and second-order closures are shown to be an accurate approach. Related work such as [34] suggests again that a RSM namely Explicit Algebraic Reynolds Stress Model (EARSM) is a good alternative to Algebraic Reynolds Stress Model (ARSM). This is because Reynolds stresses are (explicitly) linked to the mean flow velocity field, benefiting from its numerical robustness. However, as in [33], the axial velocity profile is not as accurate as the RSM configuration that we developed in the present paper, by means of a Linear Pressure Strain, periodic simulation and a very fine mesh.

Another interesting way to simulate flows with rotation is the Structure-Based Model (SBM), as seen in [35], which is a good method to avoid the relaminarisation of the flow [36]. Despite this method is able to produce axial and azimuthal profiles of a swirling flow in a rotating pipe similarly to RSM, this approach also overpredicts turbulent kinetic energy. As shown in [35] for Reynolds number $R e=20000$ and $S=0.5$, the azimuthal velocity profile is more accurately simulated by means of RSM, and the axial velocity profiles using both methods are almost identical. In these works shown in $[33,34,35]$, which are some of the first applications of RSM to rotating pipes, the important effect of the wall to RSM in confined flows was mentioned, but not investigated [37, 38]. The effect of different combinations of wall treatments and pressure-strain modelling is relevant as will be shown in Section 2.

In [27], computational studies are given of several types of rotating and swirling flows for a range of Reynolds numbers and swirl intensities aimed at identifying features that may require special attention in turbulence modelling. Results obtained by using several turbulence models concluded that $k-\epsilon$ models are not appropriate to simulate swirling flows, instead RSM is preferable. On the other hand, comparisons of Re-Normalization Group RNG $k-\epsilon$ model and RSM [29] concluded that their effects on modelling the developing turbulent swirling flow inside a straight pipe are the same as before. RSM work reported in [31] demonstrated that the turbulent swirling decay pipe flow is in good agreement with results from analytical and computational studies, with the latter implemented in FLUENT. Other successful applications include RSM on swirling flows within two concentric cylinders [30], numerical flow prediction in toroidal reactors with swirl using FLUENT [39], and turbulent flows in cyclones suffering high swirl intensity modelled by using several turbulence models [40]. For higher fidelity simulations, Direct Numerical Simulations (DNS) of swirling flows in pipes can be found in [41, 42, 43] and Large Eddy Simulations (LES) studies in $[44,45]$. Regarding the present paper, a description of the CFD simulation of a swirling flow in a rotating pipe and turbulence models are given in Section 2.

After the generation of the swirling flow, once it is issued from the pipe, it impacts on a flat plate for heat transfer purposes. The improvement of the heat transfer due to impinging jets has been vastly addressed in the literature. There is a large amount of work on non-swirling impinging jets $[46,47,48,49,50]$, and it has been demonstrated in both experimental [12, 20, 11] and computational $[13,6,21]$ studies that the addition of swirl to these jets can increase the heat 
transfer.

The swirl for impinging jets can be generated by different ways, such as by means of ducts with inner spiral trails (also referred to as helical generators or swirling strips), angled blades at the exit, agitation by stirrer blades, tangential jets to impart the swirl or by rotating pipes. Some closely related work, used for comparison in Section 3, are discussed in the following. In [11] and [20] the swirl is generated by pipes with spirals and the swirl number is based on geometrical parameters as in [51], being the swirl independent of the Reynolds number. Despite the swirl devices are similar in both papers, in [20] the hub was extended with a conical tip in order to avoid a sudden expansion, that is affecting the jet and then producing such a different behaviour in the heat transfer. Another relevant experimental work is [46]. In this paper, only non-swirling round jet flows are studied, but they are good for comparison with our non-swirling simulation, despite the Reynolds is slightly greater $(R e=23700)$. In [15] several swirl intensities are imparted by three tangential jets separated by 120 degrees.

In the present investigation, the impinging swirling jet is created by means of a rotating pipe long enough to achieve a fully-developed flow at its exit. This method of inducing swirl has the advantage of modifying the swirl intensity without varying the flow rate, i.e., swirl and flow rate are uncoupled. At the pipe inlet, the velocity field is assumed to be uniform with an imposed flow-rate corresponding to a Reynolds number $R e=23000$. The jet issues downstream from the pipe (nozzle) and impinges on the heated flat plate located at a dimensionless distance $H / D$, where $H$ stands for the nozzle to plate distance, and $D$ is the diameter of the pipe.

The proposed novel method is to split the entire simulation into two separated ones (see Figure 1): Simulation 1, for the generation of a swirling jet by means of a rotating pipe; and Simulation 2, for the simulation of both the impingement of the swirling jet on a heated plate and the heat transferred from the plate to the jet. Both simulations are described in Section 2, together with the grid convergence and validation studies. The coupling of these two simulations and the results are described and discussed in Section 3. Comparison of these results and data from the literature using similar governing parameters is included. Finally, Section 4 concludes the work.

\section{Setting-Up the CFD Simulations}

One of the greatest challenges in this computational simulation lies in the difficulty to simulate a complex large computational domain with two different stages. The choice of the turbulence model plays an important role in any CFD application. The same turbulence model can be adequate to model the swirling flow generated by the rotation of the pipe, but fail to model turbulence in the heat transfer by impingement, and vice versa. The decisions made to efficiently simulate the scenario will be described next.

\subsection{Simulation 1: CFD Simulations of a Fully-Developed Swirling Tur- bulent Flow in a Rotating Pipe.}

As described in the Introduction section, the swirling jet is to be produced in a separated simulation of a rotating pipe (Simulation 1), with a uniform non-swirling flow as inlet, in order to produce its 


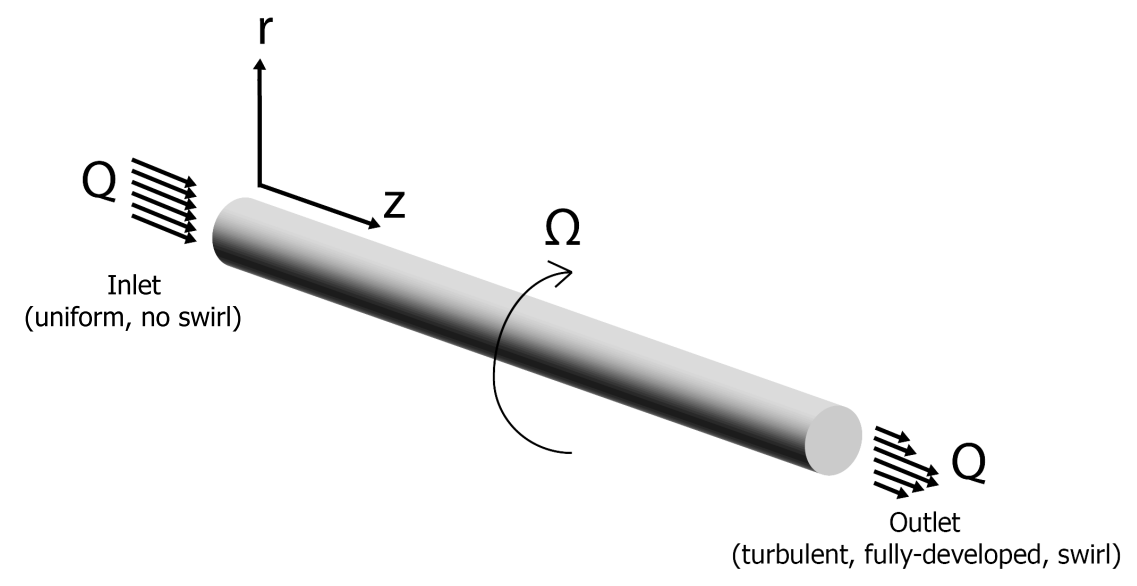

Figure 2: Sketch of the swirling flow generator by a rotating pipe (Simulation 1). The flow enters the pipe with a uniform profile and without swirl, and leaves the pipe as a swirling turbulent fully-developed jet.

swirl by the rotation of the pipe. The system is depicted in Figure 2. A 2D axisymmetric RANS simulation is implemented in FLUENT 15.0 for the present study.

The flow coming out of the pipe requires to be fully-developed, which can only be achieved with a pipe of sufficient length. In order to avoid an expensive computation, a pipe of length $L(=D)$ is simulated (see Figure 3) subject to periodic boundary conditions by imposing the mass-flow rate according to the required Reynolds number $R e=\frac{4 Q}{\pi D \nu}=23000$, Prandtl number $\operatorname{Pr}=\frac{\nu}{\alpha}=0.71$ and under rotating conditions given by the Swirl number $S=\frac{\Omega D}{2 U}=\frac{\pi D^{3} \Omega}{8 Q}$ defined as the ratio between the azimuthal velocity at the pipe wall and the mean velocity $U$, where $\nu$ is the kinematic viscosity of the fluid, $Q$ the volume-flow rate, $\alpha$ is the thermal diffusivity, $D$ the diameter of the pipe and $\Omega$ the angular velocity of rotation of the pipe.

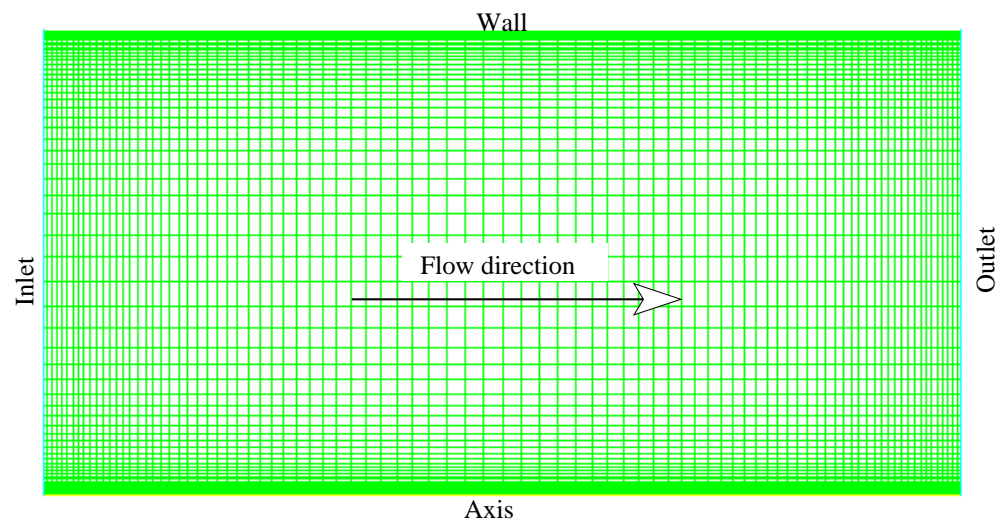

Figure 3: Piece of pipe mesh with the type of used boundary conditions used in FLUENT.

Due to the conservation of mass, at any section of the pipe:

$$
Q_{i}=Q_{o}=Q
$$


with $Q$ the volume-flow rate, which is the same at the inflow and outflow of the considered piece of pipe $\left(Q_{i}\right.$ and $Q_{o}$, respectively). In a pipe with constant diameter $D$, the pressure loss $\Delta p$ per unit length $L$ as result of friction can be modelled by the Darcy-Weisbach equation

$$
\frac{\Delta p}{L}=\frac{\lambda \rho v_{z}^{2}}{2 D}
$$

where $\frac{\Delta p}{L}$ units are $\mathrm{Pa} / \mathrm{m}$, and $\lambda$ is the friction factor, which can be calculated by different approximations [52, 53], with strong Re dependence. Since the pressure loss per unit length is constant for our fully-developed flow confined in a pipe of constant diameter $D$ (that is, this is not dependent on the axial coordinate), there exists an alternative and efficient solution to the full computational simulation of the pipe. It consists of simulating a piece of pipe, using the outflow as inflow boundary condition until a converged value of the pressure loss and the mass-flow is achieved. This is the periodic boundary condition.

While velocity components are periodic spatially, pressure is not. However, the pressure loss is constant. To solve the problem as periodic, for pressure-based solvers the pressure equation is obtained from both continuity and momentum equations. This permits a coupling of the velocity field with a correction from the pressure field, satisfying the continuity equation within an iterative scheme [54]. Specifically

$$
\nabla p(\mathbf{x})=\gamma|\mathbf{x}|+\nabla \bar{p}(\mathbf{x}),
$$

where $\nabla p(\mathbf{x})$ is the local pressure gradient with $\mathbf{x}$ the position vector, which is decomposed in the linearly-varying component, $\gamma|\mathbf{x}|$, and the gradient of the periodic component, $\nabla \bar{p}(\mathbf{x})$. Since the term $\gamma$ is not known, the iterative process in the pressure correction step takes place to calculate its value until the specified mass-flow rate is achieved computationally. Regarding the axis boundary condition, this refers to imposing axial symmetry (with zero flux of all quantities across the symmetry boundary). Since the pipe is rotating, the wall boundary condition is modelled as a moving wall imposed with a specific rotational velocity relative to the adjacent cell zone and no slip condition.

When a pipe is under rotation $(\Omega>0)$, the mean velocity of the fully-developed flow can be expressed as $\overline{\mathbf{v}}=v_{z}(r) \widehat{z}+v_{t}(r) \widehat{\theta}$, with $\widehat{z}$ and $\widehat{\theta}$ the unitary vectors in the axial and azimuthal direction, respectively. Thus, the problem can be modelled by the RANS equations in cylindrical coordinates [33] as

$$
\begin{gathered}
-\frac{v_{t}^{2}}{r}=-\frac{\partial p}{\partial r}-\frac{d \tau_{r r}}{d r}-\frac{1}{r}\left(\tau_{r r}-\tau_{\theta \theta}\right)+2 \Omega v_{t}, \\
\nu\left(\nabla^{2} v_{t}-\frac{v_{t}}{r^{2}}\right)-\frac{d \tau_{r \theta}}{d r}-\frac{2}{r} \tau_{r \theta}=0, \\
-\frac{\partial p}{\partial z}+\nu \nabla^{2} v_{z}-\frac{d \tau_{r z}}{d r}-\frac{1}{r} \tau_{r z}=0
\end{gathered}
$$

where $\tau_{i j}=\overline{v_{i}^{\prime} v_{j}^{\prime}}$ are the Reynolds shear stresses of the Reynolds stress tensor in compact notation, being $v_{i}^{\prime}$ and $v_{j}^{\prime}$ components of the fluctuation velocity $\mathbf{v}^{\prime}=v_{z}^{\prime} \widehat{z}+v_{r}^{\prime} \widehat{r}+v_{t}^{\prime} \widehat{\theta}$. 
These equations are solved numerically by using the CFD software FLUENT in a 2D axisymmetric domain. Figure 3 shows the computational mesh which corresponds to $\left[n_{r} \times n_{z}\right]=68 \times 450$ cells mesh, with $n_{r}$ and $n_{z}$ the number of computational cells in the $r$ and $z$ direction. The decision on simulating a 2D domain is based on the fact that in a fully-developed swirling flow confined in a pipe, it is expected symmetry about the central axis. As cited in [33], no symmetry-breaking bifurcation has ever been found in previous experimental or computational studies.

In CFD applications it is recommended to adequately test several different turbulence models, since there is not a single turbulence model suitable for all scenarios. For the turbulence models used in this work, which were preliminary studied in [55], the friction factor $(\lambda)$, turbulent intensity and both axial $\left(v_{z}\right)$ and azimuthal $\left(v_{t}\right)$ dimensionless velocity profiles have been validated against experimental results provided by Imao et al. [25] as depicted in Figures 4-7. The parameters used are the same as those in Imao et al., i.e. $R e=20000$ and $S=1$. For the sake of clarification, the turbulent models tested and their configurations are summarised in Table 1.

\begin{tabular}{cccc} 
Model & Options & Wall treatment & Short name \\
\hline$k-\epsilon$ & Realizable & Enhanced Wall Treatment & k-epsilon real. enh. \\
\hline \multirow{2}{*}{$k-\omega$} & SST & Not available & k-omega sst \\
& Standard & Not available & k-omega std \\
\hline \multirow{4}{*}{ RSM } & Linear Pressure-Strain & Scalable Wall Function & RSM linear sc. \\
& Linear Pressure-Strain & Enhanced Wall Treatment & RSM linear enh. \\
& Linear Pressure-Strain & Standard Wall Function & RSM linear st. \\
& Linear Pressure-Strain & Non-Equilibrium Wall Functions & RSM linear neq. \\
& Quadratic Pressure-Strain & Scalable Wall Functions & RSM quadratic sc. \\
& Quadratic Pressure-Strain & Standard Wall Functions & RSM quadratic st. \\
& Quadratic Pressure-Strain & Non-Equilibrium Wall Functions & RSM quadratic neq. \\
\hline
\end{tabular}

Table 1: Summary of the different turbulent models used.

Regarding turbulence modelling, the $k-\omega$ and $k-\epsilon$ turbulent models have been the commonplace in CFD RANS simulations, with very good performance in many problems, and there is an enormous literature with practical applications. But in certain situations, these models cannot accurately model turbulence. The simulation of a confined swirling flow is a particular application where standard eddy viscosity turbulence models fail, since these do not take into account the anisotropy of the turbulence, as discussed in Section 1. This can be actually observed in Eq. (5), since as evidenced by other authors [33], the azimuthal velocity $v_{t}$ is dependent on the Reynolds shear stress $\tau_{r \theta}$. Thus, since in these eddy viscosity models the shear stress is $\tau_{r \theta}$ has zero value, then it is impossible to physically model $v_{t}$ [33]. This is based on the modelling of the Reynolds stress tensor, which is represented by the Boussinesq assumption in compact notation as

$$
\tau_{i j}=2 \nu_{T} S_{i j}-\frac{2}{3} k \delta_{i j},
$$

being $S_{i j}$ the components of the mean strain rate tensor, $\nu_{T}$ the eddy viscosity, $k$ the turbulent kinetic energy $k=\frac{1}{2} \overline{v_{i}^{\prime} v_{i}^{\prime}}$ and $\delta_{i j}$ the Kronecker delta.

In our simulations, it is clearly seen that the $k-\epsilon$ and $k-\omega$ models do not match the axial velocity profile from experimental data, see Figure 4 (a), and the numerical azimuthal velocity 

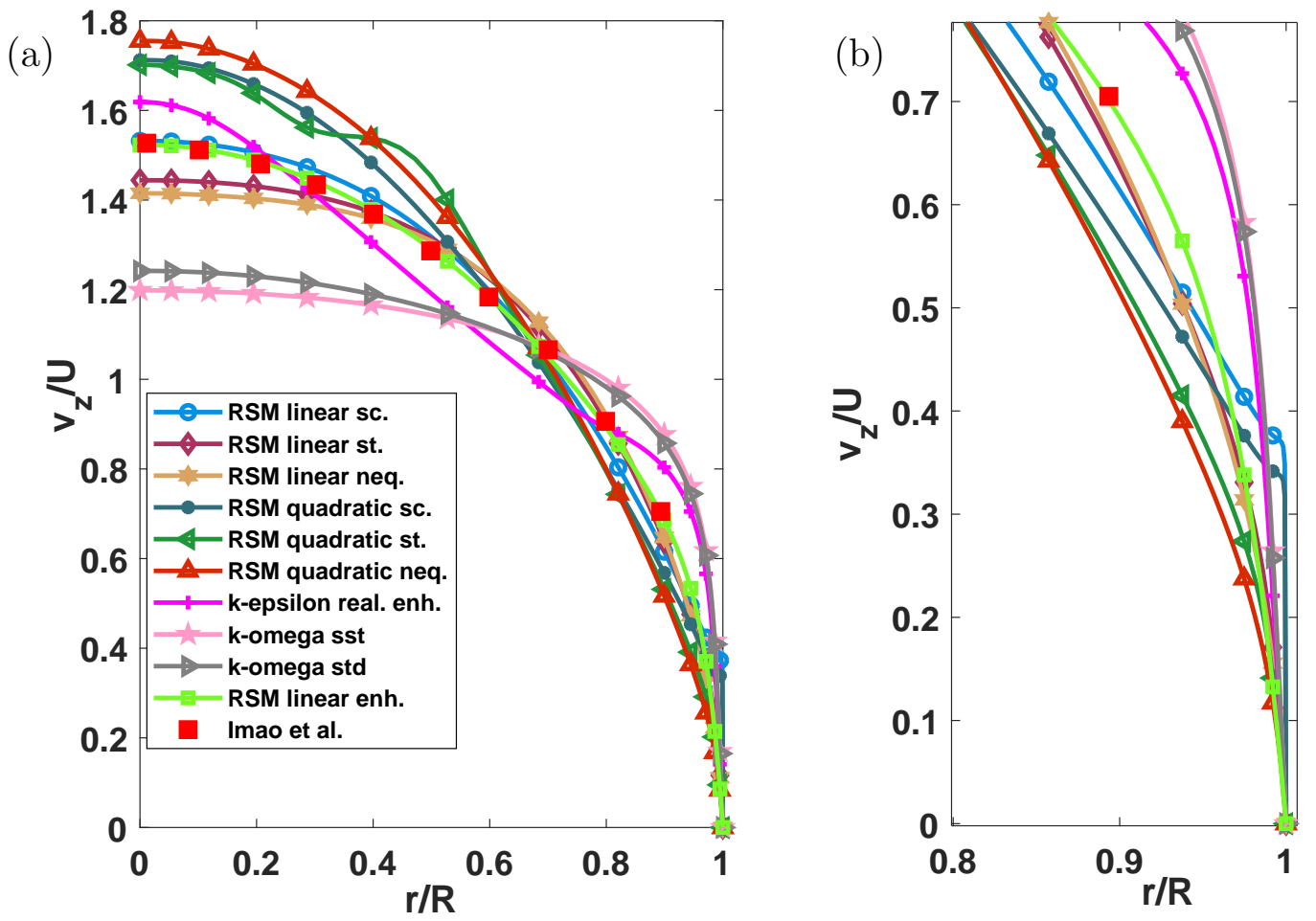

Figure 4: (a) Plot of the axial velocity profiles of the fully-developed turbulent swirling flow for several turbulence models and validation with Imao et al. [25]. (b) Zoom on the boundary layer. $R e=20000$ and $S=1$.

profile follows a solid rigid body rotation instead of a parabolic-like profile, see Figure 5 (a). Since it is known that RSM is a recommended option for swirling flows available in FLUENT (as commented in Section 1), the said $k-\epsilon$ modified models were not considered in this work. RSM includes the effect of anisotropy of turbulence, providing a superior performance with respect to eddy viscosity models in the presence of swirl. As aforementioned for eddy viscosity models, to obtain $v_{t}$ it is important to calculate the non-zero value of $\tau_{r \theta}$, to take into account rotation. The Reynolds Stress Model provides the following transport equations for the Reynolds stresses in compact notation according to [54]:

$$
\underbrace{\frac{\partial}{\partial t}\left(\rho \overline{v_{i}^{\prime} v_{j}^{\prime}}\right)}_{\text {time derivative }}+\underbrace{\frac{\partial}{\partial x_{k}}\left(\rho \overline{v_{k}} \overline{v_{i}^{\prime} v_{j}^{\prime}}\right)}_{C_{i j} \equiv \text { Convection }}=\underbrace{-\frac{\partial}{\partial x_{k}}\left[\rho \overline{v_{i}^{\prime} v_{j}^{\prime} v_{k}^{\prime}}+\overline{p\left(\delta_{k j} v_{i}^{\prime}+\delta_{i k} v_{j}^{\prime}\right)}\right]}_{D_{T, i j} \equiv \text { Turbulent Diffusion }}+
$$

$$
\underbrace{\frac{\partial}{\partial x_{k}}\left[\mu \frac{\partial}{\partial x_{k}}\left(\overline{v_{i}^{\prime} v_{j}^{\prime}}\right)\right]}_{D_{L, i j}} \underbrace{-\rho\left(\overline{v_{i}^{\prime} v_{k}^{\prime}} \frac{\partial v_{j}}{\partial x_{k}}+\overline{v_{j}^{\prime} v_{k}^{\prime}} \frac{\partial v_{i}}{\partial x_{k}}\right)}_{P_{i j} \equiv \text { Stress production }} \underbrace{\underbrace{-\rho \beta_{t}\left(g_{i} \overline{v_{j}^{\prime} \theta_{t}}+g_{j} \overline{v_{i}^{\prime} \theta_{t}}\right)}_{\equiv \text { Buoyancy production }}+}_{G_{i j}}+
$$

$$
\underbrace{\overline{p\left(\frac{\partial v_{i}^{\prime}}{\partial x_{j}}+\frac{\partial v_{j}^{\prime}}{\partial x_{i}}\right)}}_{\phi_{i j}} \quad \underbrace{-2 \mu \frac{\partial v_{i}^{\prime} \frac{\partial v_{j}^{\prime}}{\partial x_{k}} \frac{\partial x_{k}}{\partial s s i p a t i o n}}{D_{\text {Dij }}}}_{\Delta_{i j} \text { Pressure strain }} \quad F_{i j} \equiv \underbrace{-2 \rho \Omega_{k}\left(\overline{v_{j}^{\prime} v_{m}^{\prime} \epsilon_{j k m}}\right),}_{\text {Production by system rotation }}
$$



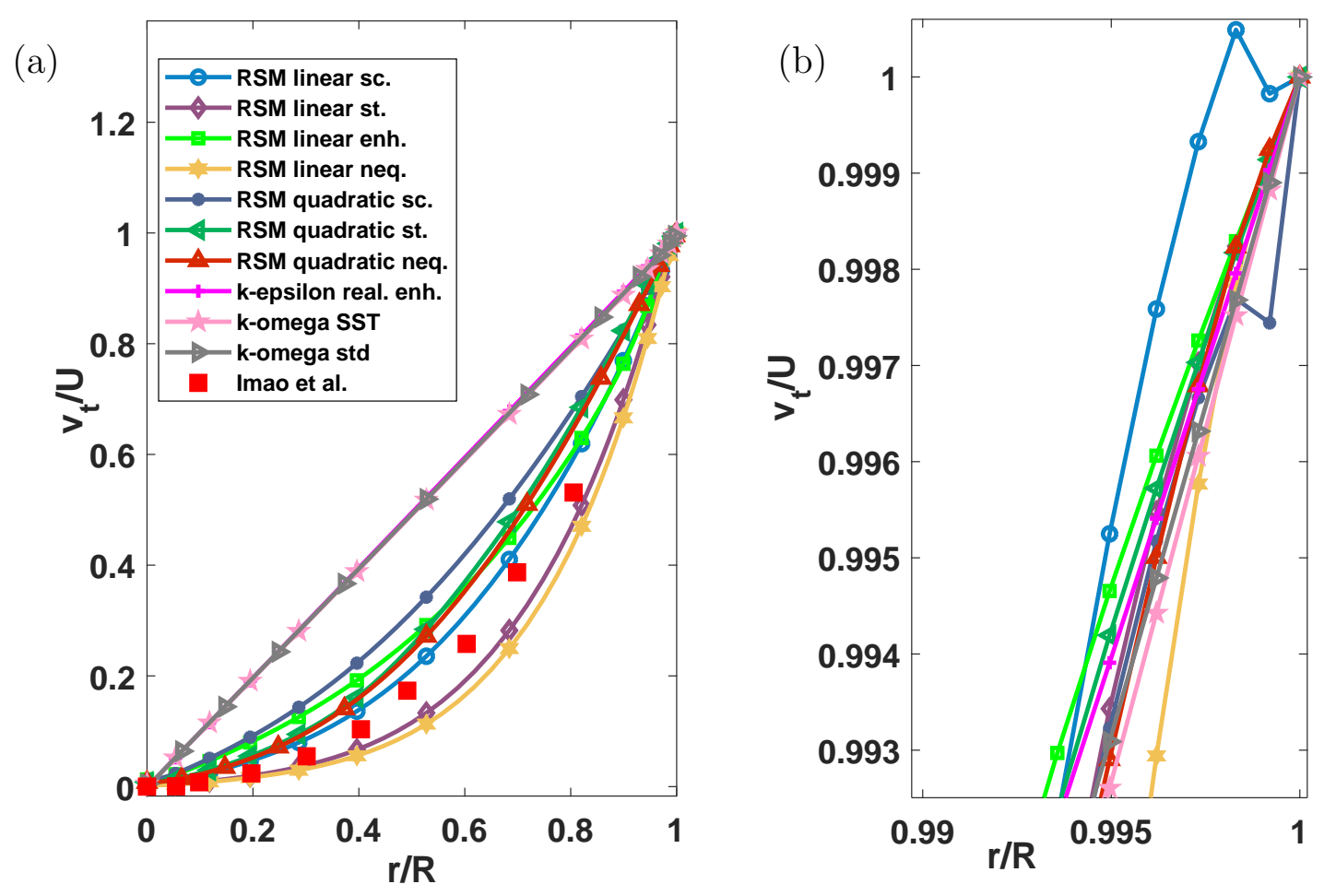

Figure 5: (a) Plot of the azimuthal velocity profiles of the fully-developed turbulent swirling flow for several turbulence models and validation with Imao et al. [25]. (b) Zoom on the boundary layer. $R e=20000$ and $S=1$

where $\Omega_{k}$ is the angular velocity of the reference frame, $\epsilon_{j k m}$ is the Levi-Civita symbol (a permutation factor), $g_{i}$ and $g_{j}$ are the components of the gravitational vectors in the $i$ th and $j$ th direction, $\theta_{t}$ is the temperature and $\beta_{t}$ is the thermal expansion coefficient. The explicit dependence on the rotation in the $F_{i j}$ term shows that this model accounts the strong swirl dependence on both axial and azimuthal velocities.

Additionally, as various terms in Eq. (8), $C_{i j}, D_{L, i j}, P_{i j}$, and $F_{i j}$ do not require any modelling but, $D_{T, i j}, G_{i j}, \phi_{i j}$, and $\epsilon_{i j}$ do, the accuracy of the RSM is limited by these closure modelling [29]. Particularly, the modelling of the pressure-strain and dissipation-rate terms is challenging, and often considered to be responsible for compromising the accuracy of RSM predictions [56]. The pressure-strain term $\phi_{i j}$ modelling is one of the key aspects in this work. To that end, several options are available:

- The Linear Pressure-Strain (LPS) option refers to modelling the pressure strain-term as a linear sum of a slow-pressure strain $\left(\phi_{i j, 1}\right)$, a rapid-pressure strain $\left(\phi_{i j, 2}\right)$ and a wall-reflection term $\left(\phi_{i j, w}\right)$, as follows [54]:

$$
\phi_{i j}=\phi_{i j, 1}+\phi_{i j, 2}+\phi_{i j, w}
$$




$$
\begin{gathered}
\phi_{i j, 1} \equiv-C_{1} \rho \frac{\epsilon}{k}\left[\overline{v_{i}^{\prime} v_{j}^{\prime}}-\frac{2}{3} \delta_{i j} k\right], \\
\phi_{i j, 2} \equiv-C_{2}\left[\left(P_{i j}+F_{i j}+G_{i j}-C_{i j}\right)-\frac{2}{3} \delta_{i j} \frac{1}{2}\left(P_{k k}+G_{k k}-C_{k k}\right)\right], \\
\phi_{i j, w} \equiv C_{1}^{\prime} \frac{\epsilon}{k}\left(\overline{v_{k}^{\prime} v_{m}^{\prime}} n_{k}^{*} n_{m}^{*} \delta_{i j}-\frac{3}{2} \overline{v_{i}^{\prime} v_{k}^{\prime}} n_{j}^{*} n_{k}^{*}-\frac{3}{2} \overline{v_{j}^{\prime} v_{k}^{\prime}} n_{i}^{*} n_{k}^{*}\right) \frac{C_{\ell} k^{3 / 2}}{\epsilon d} \\
+C_{2}^{\prime}\left(\phi_{k m, 2} n_{k}^{*} n_{m}^{*} \delta_{i j}-\frac{3}{2} \phi_{i k, 2} n_{j}^{*} n_{k}^{*}-\frac{3}{2} \phi_{j k, 2} n_{i}^{*} n_{k}^{*}\right) \frac{C_{\ell} k^{3 / 2}}{\epsilon d},
\end{gathered}
$$

with $C_{1}=1.8, C_{2}=0.60, C_{1}^{\prime}=0.5$ and $C_{2}^{\prime}=0.3$ the values of our constants in the simulations (default values in FLUENT, valid for a wide range of flows), $n_{i}^{*}$ the $i$ th component of the unit vector normal to the wall, $d$ is the normal distance to the wall, $\epsilon$ the turbulent dissipation rate, and $C_{\ell}=C_{\mu}^{3 / 4} / \kappa$, where $C_{\mu}=0.09$ and $\kappa=0.4187$, being $\kappa$ the von Karman constant.

- The Quadratic Pressure-Strain (QPS) option refers to an improved modelling of the pressurestrain that does not require a correction to account for the wall-reflection effect to obtain a satisfactory solution in the logarithmic region of a turbulent boundary layer. It must be noted that the quadratic pressure-strain model is not available when the enhanced wall treatment is selected [54].

In the CFD software, according to [54] the QPS is modelled as

$$
\begin{aligned}
& \phi_{i j}=-\left(C_{A} \rho \epsilon+C_{A}^{*} P\right)+C_{B} \rho \epsilon\left(b_{i k} b_{k j}-\frac{1}{3} b_{m n} b_{m n} \delta_{i j}\right)+\left(C_{C}-C_{C}^{*} \sqrt{b_{i j} b_{i j}}\right) \rho k S_{i j} \\
& +C_{D} \rho k\left(b_{i k} S_{j k}+b_{j k} S_{i k}-\frac{2}{3} b_{m n} S_{m n} \delta_{i j}\right)+C_{E} \rho k\left(b_{i k} \Omega_{j k}+b_{j k} \Omega_{i k}\right),
\end{aligned}
$$

where $b_{i j}$ is the Reynolds-stress anisotropy tensor

$$
b_{i j}=-\left(\frac{-\rho \overline{v_{i}^{\prime} v_{j}^{\prime}}+\frac{2}{3} \rho k \delta_{i j}}{2 \rho k}\right)
$$

$\Omega_{i j}$ is the mean rate-of-rotation tensor

$$
\Omega_{i j}=\frac{1}{2}\left(\frac{\partial v_{i}}{\partial x_{j}}-\frac{\partial v_{j}}{\partial x_{i}}\right),
$$

and all the constants used with this model in our simulations are set to $C_{A}=3.4, C_{A}^{*}=1.8$, $C_{B}=4.2, C_{C}=0.8, C_{C}^{*}=1.3, C_{D}=1.25$ and $C_{E}=0.4$. These are the default values in FLUENT, valid for a wide range of flows.

In order to solve the boundary layer accurately, the effect of the rotating walls in Simulation 1 must be taken account. There are four options of wall treatment methods that are available for RSM as described below. 
- Standard Wall Function. This is a classic choice which works for many flows. The logarithmic law (log-law) for the momentum fluxes is given by

$$
\underbrace{\frac{U_{P} C_{\mu}^{1 / 4} k_{P}^{1 / 2}}{\tau_{w} / \rho}}_{U^{*}}=\frac{1}{\kappa} \ln (E \underbrace{\frac{\rho C_{\mu}^{1 / 4} k_{P}^{1 / 2} y_{P}}{\mu}}_{y^{*}}),
$$

where $y_{P}$ is the distance of the first point $\mathrm{P}$ from the wall, $U_{P}$ is the time average velocity of the flow at $\mathrm{P}, C_{\mu}=0.09$ is the constant-pressure specific heat, $k_{P}$ is the turbulent kinetic energy at the grid point $\mathrm{P}$, and $\tau_{w}$ is the shear stress at the wall on the direction of $U_{p}$ [57]. The variable $y^{*}$ is known as the wall unit, and the log-law for mean velocity is valid for $30<y^{*}<300$ [54]. In the CFD solver the log-law is used when $y^{*}>11.225$. If the computational mesh has a value of $y^{*}<11.225$ at the wall-adjacent cells the laminar stressstrain relationship $U^{*}=y^{*}$ is applied. A formal description of this wall function can be found from [57]. Further information on the relations for heat fluxes can be found from [58].

- Scalable Wall Function. When a very fine grid is used, wall functions may lead to misleading results, due to the fact that a refinement may lead to the first point in the sublayer. The scalable wall function model avoids such problem on grids with $y^{*}<11.225$. The method is simple and is basically the Standard Wall Function with the limiting factor

$$
\widetilde{y^{*}}=\max \left(y^{*}, 11.225\right)
$$

being substituted into the standard $y^{*}$. In essence, the performance on coarse grids, i.e. $y^{*}>11.225$, is identical to the those obtained by using Standard Wall Function.

- Non-Equilibrium Wall Function. This approach by [59] uses the Launder and Spalding's log-law introduced in the Standard Wall Function, but with a new formulation for the mean velocity $U_{P}$ sensitive to pressure-gradient effects, defined in FLUENT as [54]

$$
\tilde{U}=U-\frac{1}{2} \frac{d p}{d x}\left[\frac{y_{v}}{\rho \kappa \sqrt{k}} \ln \left(\frac{y}{y_{v}}\right)+\frac{y-y_{v}}{\rho \kappa \sqrt{k}}+\frac{y_{v}^{2}}{\mu}\right],
$$

where $\kappa$ is the von Karman constant and $y_{v}$ is the physical viscous sublayer thickness

$$
y_{v} \equiv \frac{11.225 \mu}{\rho C_{\mu}^{1 / 4} k_{P}^{1 / 2}} .
$$

To compute the turbulent kinetic energy for the cells neighbouring the wall, a method based on a two-layers concept (a viscous sublayer and a fully turbulent layer) is applied, as introduced in [54], where the reader is referenced for more details.

- Enhanced Wall Treatment. This method allows the combination of a two-layer modelling and the use of wall functions. If $y^{+}<1$ the viscous sublayer can be solved and a two-layer approach is applied. For larger values of $y^{+}$wall functions may be used. This is a valuable property for complex geometries. The two-layer modelling in Enhanced Wall Treatment 
consists of classifying the flow into a viscosity-affected region and a fully-turbulent region. This is computed by a turbulent Reynolds number based on the wall distance $y$

$$
\operatorname{Re}_{y} \equiv \frac{\rho y \sqrt{k}}{\mu} .
$$

If $R e_{y} \leq 200$, the region becomes viscosity-affected, and the turbulent viscosity is modelled by

$$
\mu_{t, \mathrm{visc}}=\rho C_{\mu} \ell_{\mu} \sqrt{k}
$$

with the length scale, $\ell_{\mu}$, modelled according to [60]. When $R e_{y}>200$, the region becomes a fully-turbulent region, and the turbulent viscosity is modelled by either the $k-\epsilon$ or RSM, depending on which turbulent model is used. The smooth blending of the turbulent viscosities according to [61] becomes

$$
\mu_{t, \mathrm{enh}}=\lambda_{\epsilon} \mu_{t}+\left(1-\lambda_{\epsilon}\right) \mu_{t, \mathrm{visc}},
$$

with the smooth blending function given by

$$
\lambda_{\epsilon}=\frac{1}{2}\left[1+\tanh \left(\frac{\operatorname{Re}_{y}-200}{\frac{\left|\operatorname{Re}_{y}-200\right|}{\operatorname{arctanh}(0.98)}}\right)\right] .
$$

For the Enhanced Wall Treatment to the entire wall region, laws-of-the-wall are implemented by means of the Enhanced Wall Function option. In FLUENT, this approach is based on the work in [62], where the Enhanced Thermal Wall Functions were suggested. The approach consist of merging the linear (laminar) and logarithmic (turbulent) wall laws by means of the blending function defined as

$$
\Gamma=-\frac{a_{\Gamma}\left(y^{+}\right)^{4}}{1+b_{\Gamma} y^{+}}
$$

where $a_{\Gamma}=0.01$ and $b_{\Gamma}=5$, and $y^{+}$is the dimensionless wall distance. The blending of the two wall laws is now given by the law

$$
u^{+}=e^{\Gamma} u_{\mathrm{lam}}^{+}+e^{\frac{1}{\Gamma}} u_{\mathrm{turb}}^{+},
$$

where $u^{+}$stands for the dimensionless mean near-wall velocity profile and

$$
u_{\mathrm{lam}}^{+}=y^{+}\left(1+\frac{\alpha_{p}}{2} y^{+}\right)
$$

where

$$
\alpha_{p}=\frac{\mu}{\rho^{2}\left(U^{*}\right)^{3}} \frac{d p}{d x}
$$

is the account of pressure gradients. Further modelling of the enhanced turbulent lawof-the-wall for $u_{\text {turb }}^{+}$can be found in $[63,64]$. For the Enhanced Thermal Wall Function, the blending function $\Gamma$ includes the molecular Prandtl number. For its development, the reader is suggested to see [62]. For further information and applications on Enhanced Wall Functions, the authors suggest to see [54, 65, 66]. 
For the CFD simulations of Simulation 1 using RSM, the performance is shown in Figures 4 and 5. From these figures it can be seen that RSM with QPS overestimated the velocity profiles $v_{z} / U$ and $v_{t} / U$ (legend 'RSM quadratic' and its variations). Figure 4 (a) shows that $v_{z} / U$ performed considerably worse by using LPS (legend 'RSM quadratic' and its variations). It is thought that such overestimation of velocity profiles may be sensitive to the methods used in the computation of the boundary layer, because of the interaction between the wall and the fluid leading to swirl. One can see that when LPS is applied, the profiles are always underestimated, except with the Scalable Wall Function, as depicted in Figure 4 and 5 under legend 'RSM linear sc'. The profiles in the region near to the wall, for both QPS and LPS with Scalable Wall Function, are not realistic, especially for $v_{t}$ (see Figure $5(\mathrm{~b})$, legend 'RSM linear $\mathrm{sc}^{\prime}$ ). Moreover, the QPS modelling does not include any correction for the wall-reflection effect to properly solve the logarithmic region of the turbulent boundary layer [54]. Since this term corrects the magnitude of turbulent velocity fluctuations perpendicular to the wall for a better wall treatment in RSM [67], the option was activated for LPS. These reasons could explain why LPS yields better results.

For LPS, the best wall treatment was the Enhanced Wall Function, see Figure 4 and 5, legend 'RSM linear enh'. The good performance of this treatment is not surprising since a very fine mesh $\left(y^{+}<1\right.$, with $y^{+}$the dimensionless wall distance) was generated and for such, the Enhanced Wall Function allows to resolve the viscous sublayer [68]. The results using both Standard and Non-Equilibrium Wall Functions (legends 'RSM linear st' and 'RSM linear neq', respectively) were also quite good, although the Standard Wall Functions are recommended for coarser meshes at the boundary layer and Non-Equilibrium Wall functions are of interest in applications with separation, impingement or sudden pressure changes [54].

Additionally, the normalized mean error at different radial locations between the numerical and experimental velocity components, as well as the relative error with respect to the experimental wall friction factor, are depicted in Figure 6. This figure also confirms that the best performing turbulence model is the RSM with Linear Pressure-Strain and Enhanced Wall Treatment, legend 'RSM linear enh'.

Since this swirling flow is used as impinging jet, it is also relevant to check the accuracy in the computation of turbulence, because large inaccuracies may affect in downstream flow. In RANS, eddy viscosity models are not able to compute the Reynolds stresses. However, in RSM each component of the stress tensor is available. One can then obtain the turbulent intensity by normalising with the mean velocity, $U$, the square roots of the stress components given by

$$
\begin{aligned}
& u_{s t r}^{2}=\tau_{z z}=\overline{v_{z}^{\prime} v_{z}^{\prime}}, \\
& v_{s t r}^{2}=\tau_{\theta \theta}=\overline{v_{t}^{\prime} v_{t}^{\prime}}, \\
& w_{s t r}^{2}=\tau_{r r}=\overline{v_{r}^{\prime} v_{r}^{\prime} .}
\end{aligned}
$$

These are compared with the turbulent intensity experimental results from [25] for the fullydeveloped flow, as shown in Figure 7. The simulated velocity fluctuations are quite close to the experimental data, especially in the closest distances to the wall, where the azimuthal velocity and friction effects are more relevant. Also, as in the experimental study, the velocity fluctuation in the radial component is the smallest, as a consequence of the rotation of the pipe, which tends to stabilise such feature. 


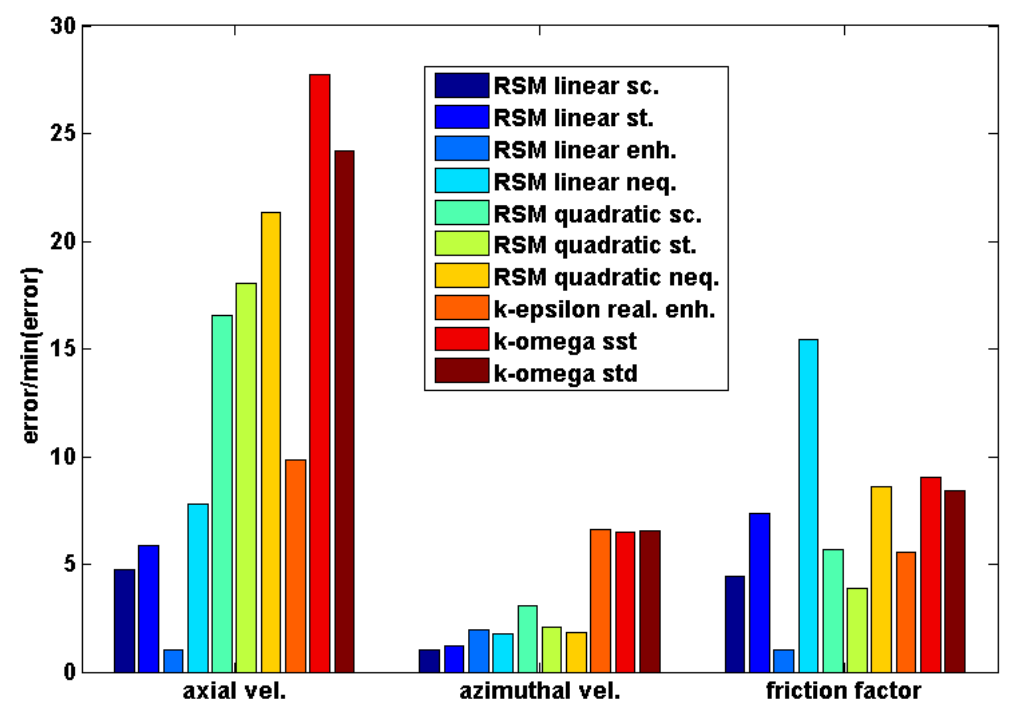

Figure 6: Plot of the errors of relevant parameters of the simulation for several turbulence models, with respect to the minimum value of the error (the RSM linear enh.). The error measure is the euclidean norm.
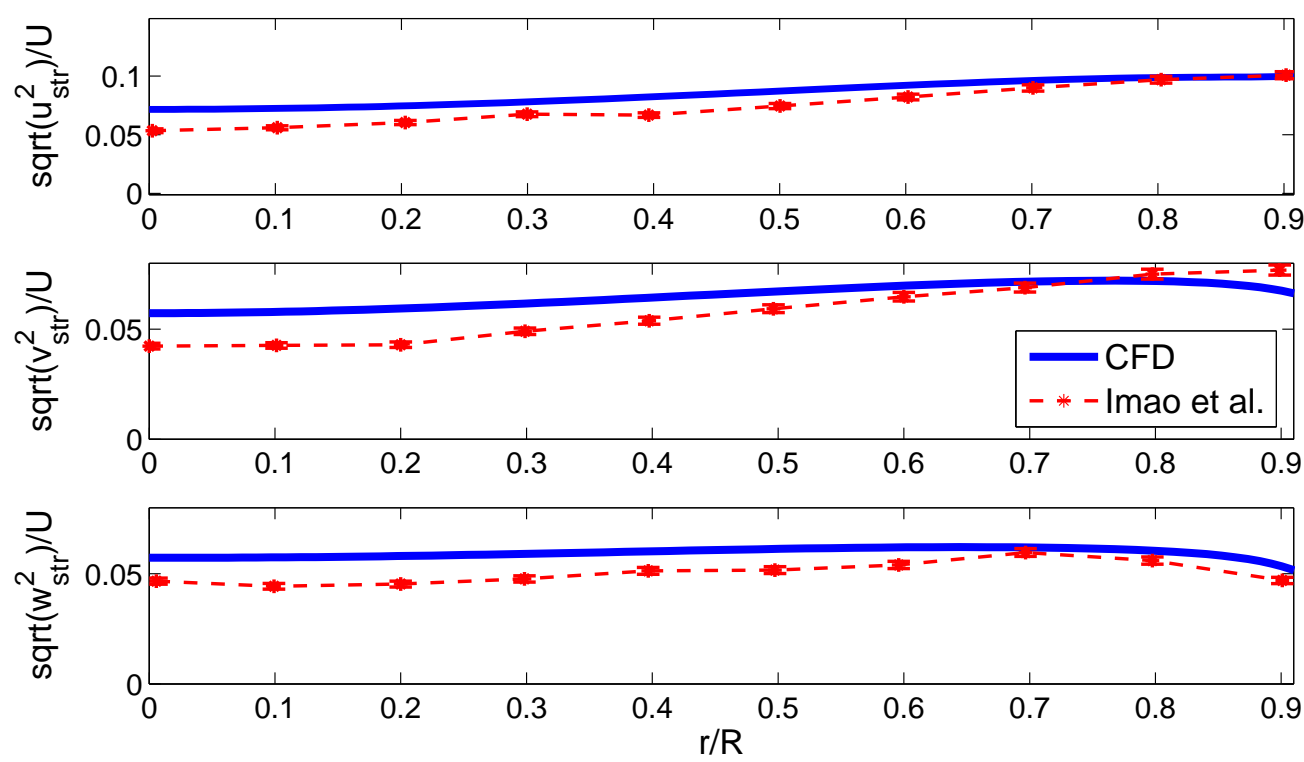

Figure 7: Comparison of computational and experimental turbulent intensities in the axial, azimuthal and radial direction. The experimental values have the $\pm 3 \%$ error bars, as reported in $[25]$.

As observed before, the turbulent model with the best performance for modelling the generation of a swirling turbulent fully-developed flow in a periodic pipe is the RSM with Linear Pressure- 
Strain and Enhanced Wall Treatment. This statement is true for similar values of $R e$ and $S$. However the performance obtained for using notably larger values of the set of parameters is not unknown. For this reason, in Figure 8 a simulation for $R e=50000$ and $S=1$ has been run and validated with data from [69] by using different turbulent models. It can be seen that, again, the best match with experimental data is obtained by the RSM with Linear Pressure-Strain and Enhanced Wall Treatment, with a very good performance at this higher value of Re. At this Re, the effect of swirl is less noticeable and the curvature of $v_{t}$ is less intense.
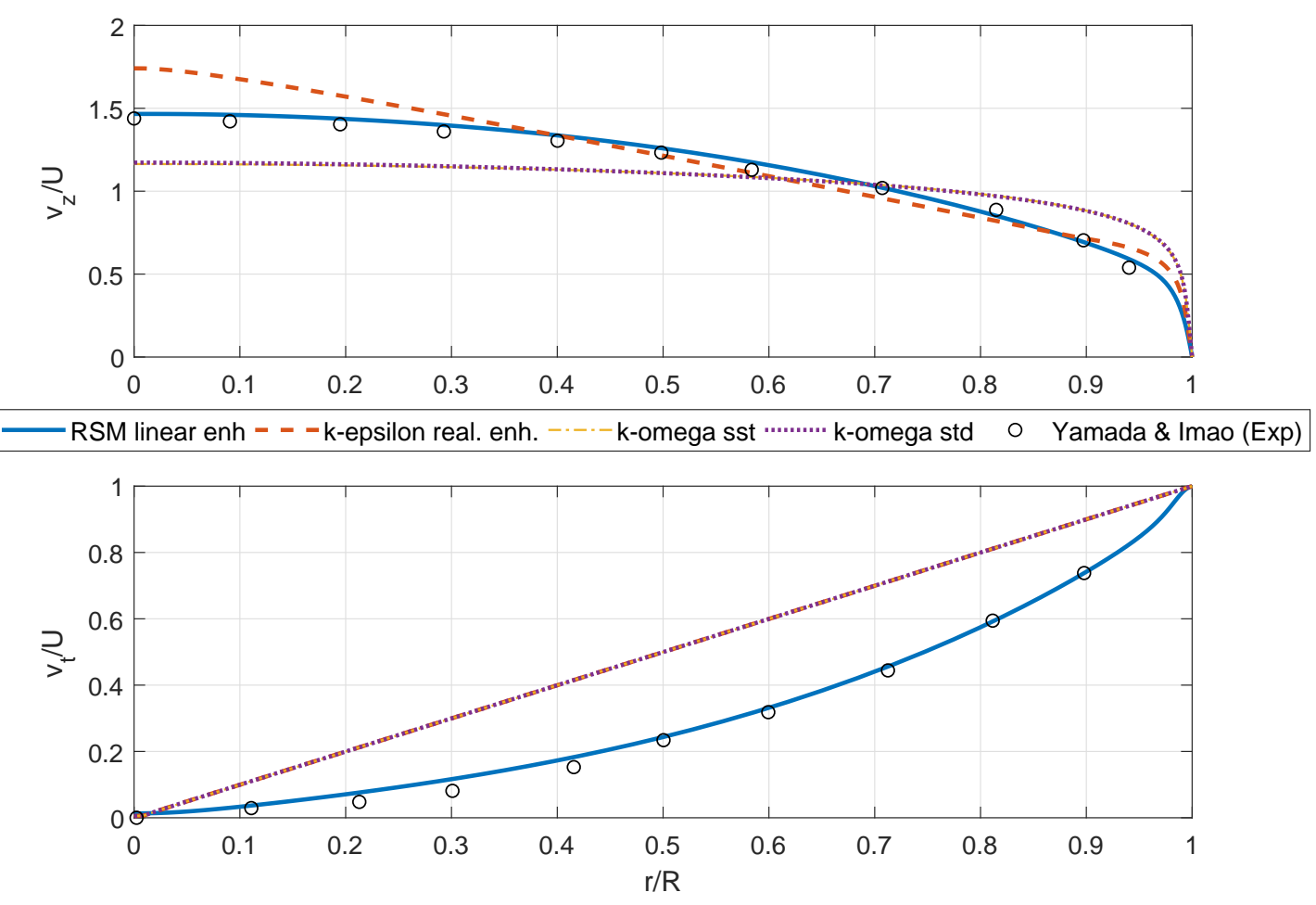

Figure 8: Axial and azimuthal velocity profiles of the fully-developed swirling flow with $R e=50000$ and $S=1$. The experimental data Yamada \& Imao (Exp) are taken from [69].

As the study in this paper concerns an incompressible flow, a pressure based formulation is used. The pressure-velocity coupling is done by SIMPLE (Semi-Implicit Method for PressureLinked Equations) algorithm [70]. The spatial discretisation of convection terms is used with Second Order Upwind methods, while the gradients are evaluated by means of the Least Squares Cell Based algorithm.

\subsection{Grid uncertainty}

The uncertainty of the grid has been analysed for Simulation 1 following the Grid Convergence Index (GCI) procedure as described in [71], which is based on Richardson extrapolation. In Simulation 1, the parameter under study is the friction factor, $\lambda$, as it is a suitable variable to check whether the boundary layer is well solved or not. The GCI analysis of $\lambda$ is shown in Figure 
9 where a discretization error of only a $0.2 \%$ is achieved with 200 iterations per minute in using the mesh with $\left[n_{r} \times n_{z}\right]=68 \times 450$ cells. The mesh ensures the first computational cell at a dimensionless wall distance of $y^{+}<1$ along the walls of the pipe. Despite Simulation 2 is described in the following section, note that the discretisation error of the mesh is not shown in this work because it has been provided in $[6,14]$ the appropriate mesh for impinging jets at similar Reynolds number, with only a $0.4 \%$ of discretisation error.

The validated results from Simulation 1 are to be used as the inlet condition for Simulation 2, which will be discussed next.

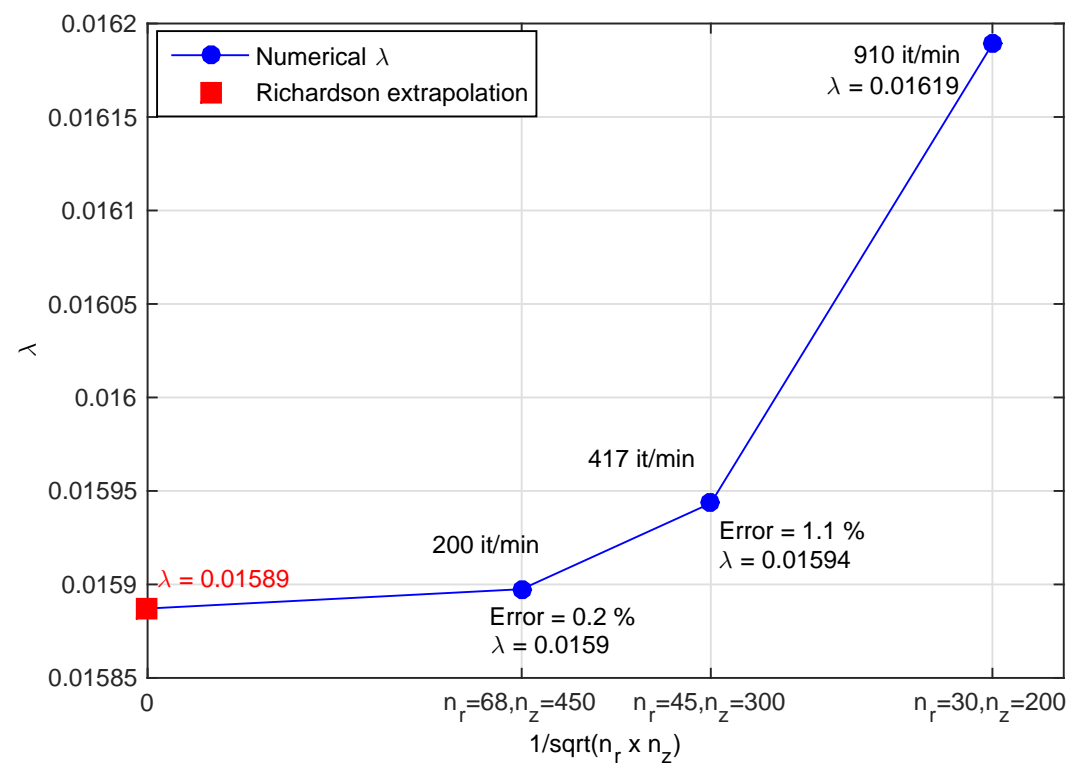

Figure 9: Grid Convergence Index to quantify the discretization errors and Richardson Extrapolation. The iterations per minute and the corresponding error are also included in the figure for the mid and fine grids.

\subsection{Simulation 2: CFD Simulations of the Heat Transfer to a Heated Solid Flat Plate from an Impinging Swirling Jet}

As mentioned in Section 1, impinging jets for heat transfer have many applications. The effectiveness of the heat transfer depends on different factors, such as the $H / D$ distance, nozzle shape, type of fluid or the surface of the plate. In this work, the configuration consists of a flat plate and the distance between the exit of the pipe and the plate is at $H / D=2$. For this nozzle-to-plate distance there is a large amount of impinging jet data to compare with.

The simulations have been conducted as 2D axisymmetric RANS using FLUENT 18.0. The property of axisymmetry in swirling free jets issuing from a round nozzle and under similar swirl intensities has been observed experimentally by several researchers. In [72], an extensive study on jets is developed evidencing this. Reynolds numbers ranging from 20000 up to 60000 are used in their work. In [73], a swirling jet at $R e=24000$, and different swirl intensities $S=0$ and $S=0.5$ 
is analysed both computational and experimentally, with evidenced axisymmetry. Additionally, in $[74,75,76]$, computational studies of 2D axisymmetric swirling impinging jets are developed.

For the CFD simulation of Simulation 2, the details of the discretised domain and the boundary conditions are depicted in Figure 10. The size of the shown grid is $\left[n_{x} \times n_{h}\right]=140 \times 250$ cells, ensuring a $y^{+}<1$ along the plate. In practice, the $x$ axis is the same as the $r$ one when the pipe was analysed, but a different notation was intended to avoid confusion between the parameters at the exit of the pipe and those on the flat plate. Similarly, $h$ is the axial coordinate with origin in the nozzle tip, and $y$ is the axial coordinate whose origin is the flat plate. A piece of the rotating pipe was also simulated in the computational domain of Simulation 2 for a less abrupt coupling of the profiles from the pipe (referred to as Wall (rotation) in Figure 10). This piece of rotating pipe in Simulation 2, is also recommendable to compute any minimum sensitivity of the pipe flow to the impingement condition.

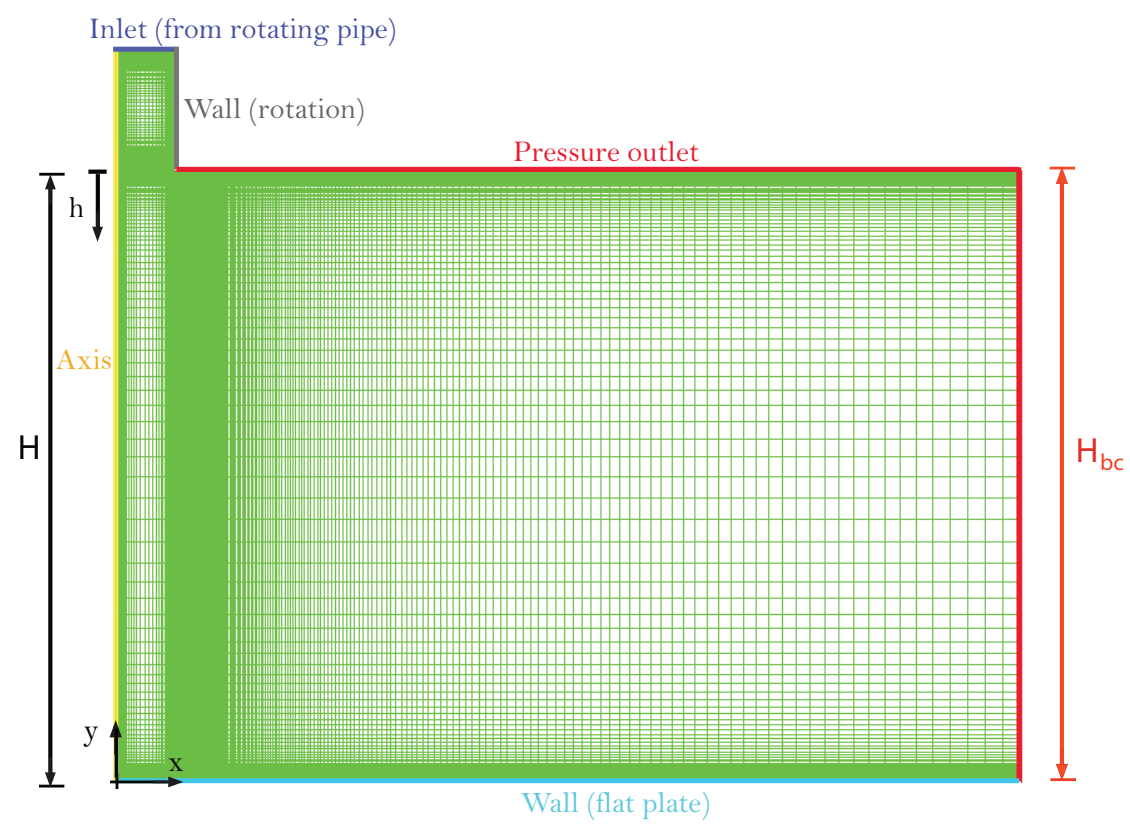

Figure 10: Computational grid and boundary conditions imposed to the CFD simulation Simulation 2. $H_{b c}$ stands for the height of the upper boundary condition, that can be different than $H$. The size of the pipe wall is kept fixed at a length $D$.

The turbulence model used in Simulation 2 is the $\operatorname{SST} k-\omega$. This choice resulted to a good performance for impinging swirling jet flows in previous work such as those described in [6] and [14]. For further information about the computational features of the simulation, including the discretisation error and the proper selection of the SST $k-\omega$ turbulence model, the authors suggest to see $[6,14]$, since the difference between these swirling jet flows is just the swirl generation mechanism. The numerical results of the present study for the different used turbulent models have been validated with experimental data of impinging jets from [11] and [46], as shown in Figure 11. The best model to match experimental data has been the SST $k-\omega$ with the Production 
Kato-Launder option, as depicted in Figure 11 under legend 'SST k- $\omega$ conf. 4'. An accurate numerical radial Nusselt number $(N u)$ along the flat plate can be observed. This option, available in FLUENT, provides a correction to the common turbulent kinetic energy overproduction that takes place as a result of high values in the shear strain rate term in situations with sudden changes in velocity as in impinging jets flows $[77,54,78]$. In Figure 12, velocity profiles on the plate are compared with experimental data from [79], with a very precise match. The RSM turbulent model was also tested, but with unsuccessful results: it was complicated to converge the simulation with swirl, which blew-up easily, and the achieved results were not realistic. In previous works [80, 81], the RSM turbulence model was used for non-swirling jet flows, yielding unreliable results too.

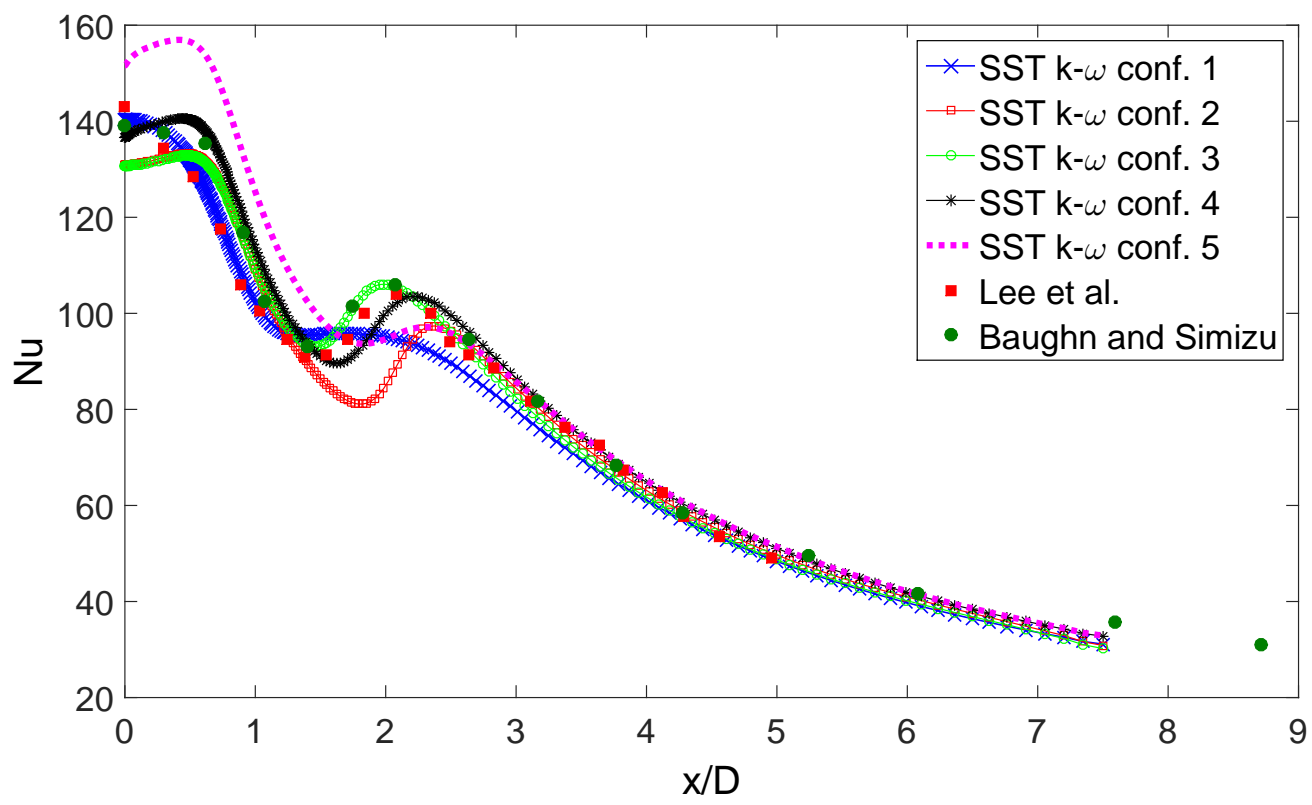

Figure 11: Validation of the turbulence model for $H / D=2$. The different configurations of the SST $k-\omega$ turbulence model correspond to conf. 1: with transitional flow (old model in FLUENT version 6.3); conf. 2: with Low Reynolds correction; conf. 3: without Low Reynolds correction; conf. 4: Transition SST with the Production Kato-Launder option; and conf. 5: Transition SST with the Production Limiter option.

A higher value of the Reynolds number, $R e=35000$, has been also simulated, whose results are depicted in Figure 13. These numerical results are validated against data obtained from [82], in which the swirling flows are generated by a different mechanism. To replicate their results and test our turbulence modelling for this higher value of $R e$, their experimental axial and azimuthal velocity profiles have been used as inlet in Simulation 2 for $S=0,0.16$ and 0.27 . Figure 13 shows that both experimental (EXP) and the simulated numerical (CFD) pressure coefficient, $C_{p}$, match very well.

Despite to study the jet flow at different Reynolds numbers would be arguably interesting, this is actually out of our scope, since we have focused our efforts only on the effect of $S$ and $H / D$ on the jet and heat transfer at $R e=23000$, because of its popularity in the literature on impinging 


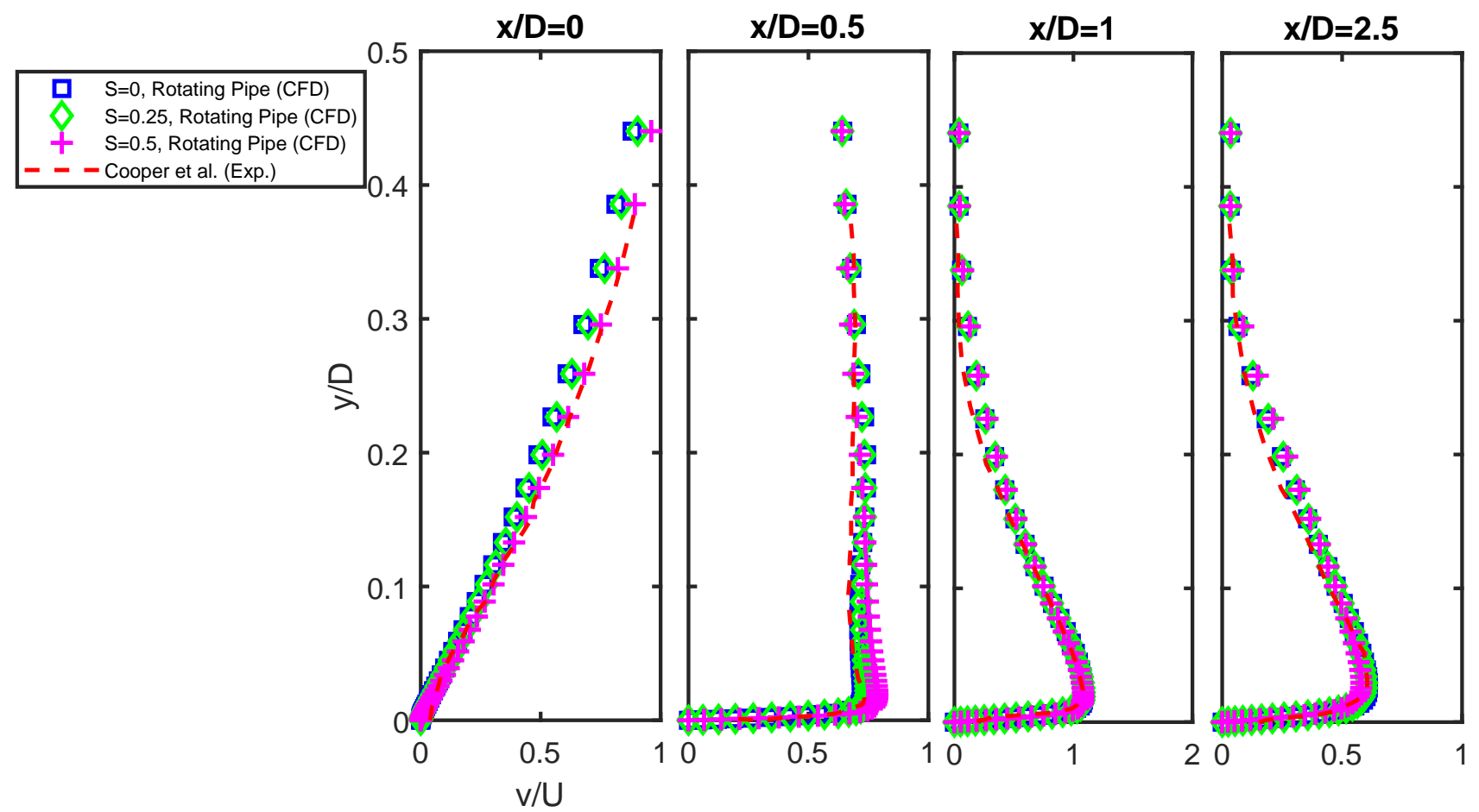

Figure 12: Comparison of velocity magnitude profiles at different $x / D$. Experimental results from [79], used for comparison in [83]. The $y / D$ coordinate represents the distance from the plate.

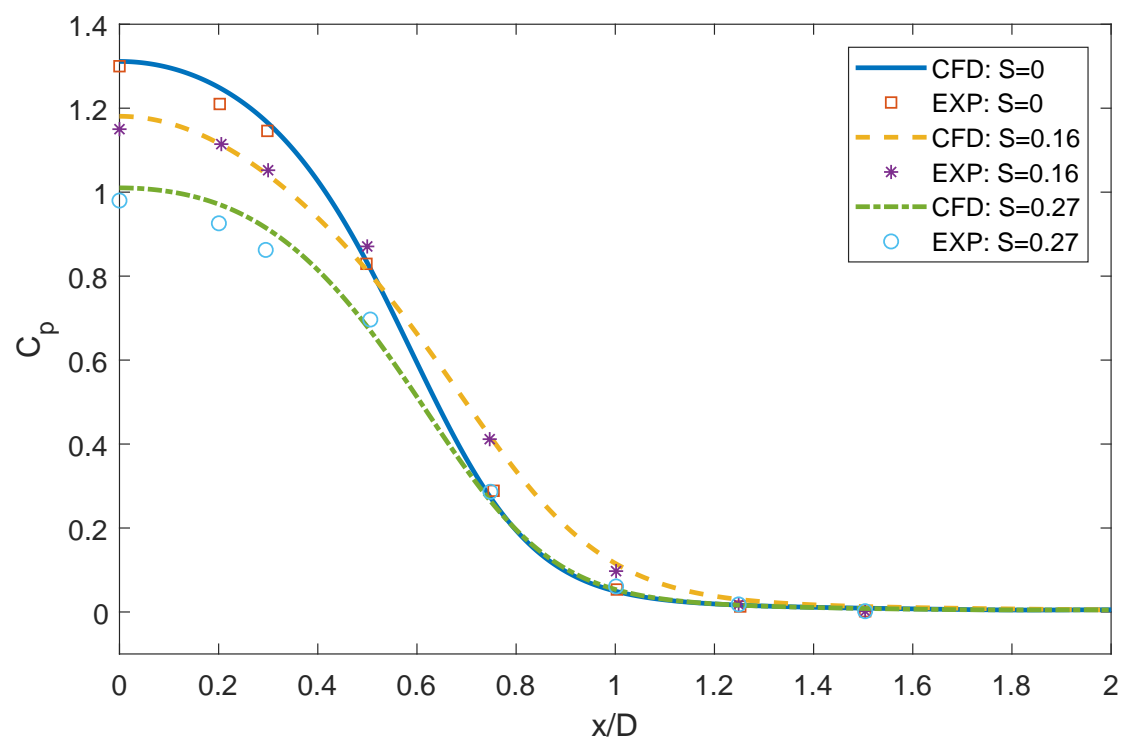

Figure 13: Pressure coefficient at $R e=35000$ and $S=0,0.16$ and 0.27 . The experimental data (EXP) are taken from [82].

jets, as seen in most citations in Section 1. Nevertheless, the results provided for both Simulation 1 and 2 at high Reynolds numbers show that the configuration for the CFD solver can be also 
valid under such conditions.

As the turbulence model used for the pipe and the one used for the impinging heat transfer problem are both different, a single simulation of the two scenarios is not adopted. To overcome this drawback, the two-step CFD simulations are coupled in the following way: first, the CFD simulations of the swirling flow confined in a rotating pipe with the RSM turbulent model is computed (Simulation 1), and second, the velocity and $k-\omega$ profiles at the exit of the pipe are imposed as inlet boundary conditions for the heat transfer simulation (Simulation 2). The turbulent parameters are taken as

$$
\begin{aligned}
& k=\frac{2}{3}(U I)^{2}, \\
& \omega=\rho \frac{k}{\mu} \beta^{-1},
\end{aligned}
$$

where $k$ is the turbulent kinetic energy, $I$ is the turbulence intensity, $\rho$ is the density of the fluid, $\omega$ is the turbulence dissipation rate and $\beta \equiv \mu_{t} / \mu$ is the turbulent viscosity ratio. The turbulent kinetic energy is available from the RSM simulations and the turbulence dissipation rate is evaluated by using Eq. (30), in order to obtain the inlet boundary condition for Simulation 2. For the other boundary conditions, the no-slip and symmetry boundary conditions are imposed at the walls and axis of symmetry, and the small piece of pipe included is also rotating at the angular velocity $\Omega$. The pressure outlet boundary condition is useful when flows are leaving a domain and the pressure is imposed at those boundaries by fixing the static pressure to a certain value (atmospheric pressure in the present case) at the outlet boundary [54]. FLUENT extrapolates flow quantities from the interior of the domain, by taking into account that velocity and other transported quantities have gradients fixed to zero value at the boundary.

For jets at higher Reynolds numbers, a pressure suction area appears because of the difference in velocities between the jet and the surrounding flow. This can affect the simulation of the jet, and thus, the numerical heat transfer. For this reason, it is interesting to see how the domain boundary conditions might affect the simulation results. The impact of the height of the upper boundary $\left(H_{b c}\right)$, as illustrated in Figure 10, is studied. In this illustration $H_{b c}=H$, but the reader must take into account that at the different positions of $H_{b c}$ the pipe exit is always at $H / D=2$. The effect is found to be negligible even for the worst-case scenario of $H / D=2$, as shown in Figure 14, where the evolution of the Nusselt number is plotted for three different values of $H_{b c}$. Since it is not relevant, the initial configuration shown in Figure 10 with $H_{b c}=H$ is kept.

\section{Discussion of Results}

In this section, the CFD simulation results are analysed for different values of $S$, in order to examine the impact of the swirl intensity. A comparison of these results with other swirl generation mechanisms from the literature is also carried out. 


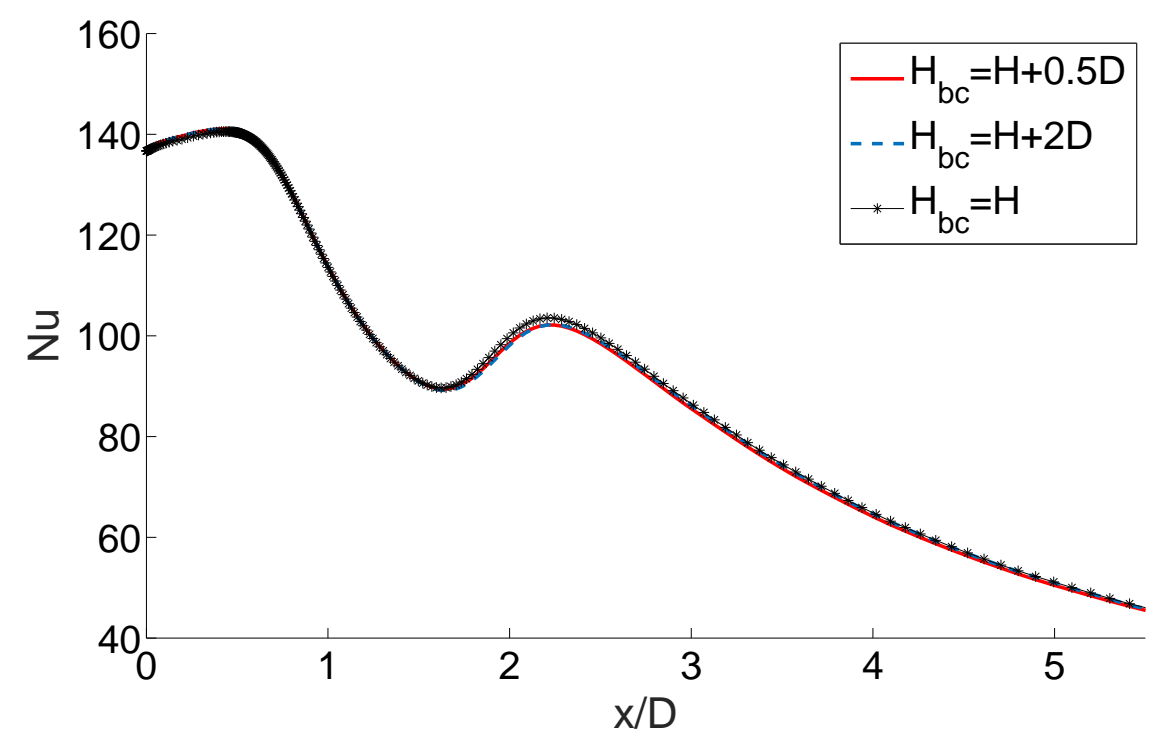

Figure 14: Effect of the position of the upper boundary condition in the Nusselt number.

\subsection{Results of the CFD simulation at Different Configurations}

For $H / D=2$ and $R e=23000$, several experimentalists have reported a secondary peak in the radial distribution of the Nusselt number [11, 46]. It is suggested that this secondary peak, which yields an M-shaped distribution, is caused by the transition from laminar to turbulent boundary layer in the wall jet as suggested by [84,85]. This transition seems to be the consequence of the disappearance of the pressure gradient which exists in the stagnation region and affects the stabilization of the flow and turbulent intensity [86]. More recently, in [10] a large number of computational LES simulations have been carried out to gain more understanding of the secondary peak, which has been found to be related with changes in the flow velocity and turbulence in the boundary layer on the plate.

Because of the said physical meaning, the appearance of the secondary peak in the present computational work is a good indicator to the reliability of the RANS simulations. As shown in Figure 15, both of the peaks in the turbulence intensity and wall shear stress on the plate coincide with the position of such peak in the radial distribution of Nusselt number. This peak cannot be obtained with $k-\omega$ when the transitional flow option is used (SST $k-\omega$ conf. 1 in Figure 11). To better comprehend the challenges in CFD for this type of problems with swirling jet flows, there is only limited work using LES for heat transfer by impinging jets [87, 9, 88, 89, 10]. The matching of numerical and experimental data for the radial Nusselt number distribution is usually poor, despite the free jet flow matches well with the experimental data $[88,9,89]$. In addition, either the secondary peak is not captured [9] or the location is not accurately computed [89]. Despite all the work done by many authors for decades, the CFD simulation of the heat transfer from impinging jets is still a challenging investigation that needs further research to answer critical questions [10]. This brings out the importance of the present investigation with the novel use of rotating pipes.

Figures 16, 18, 19 and 20 provide a better understanding on how the fluid flows along the 


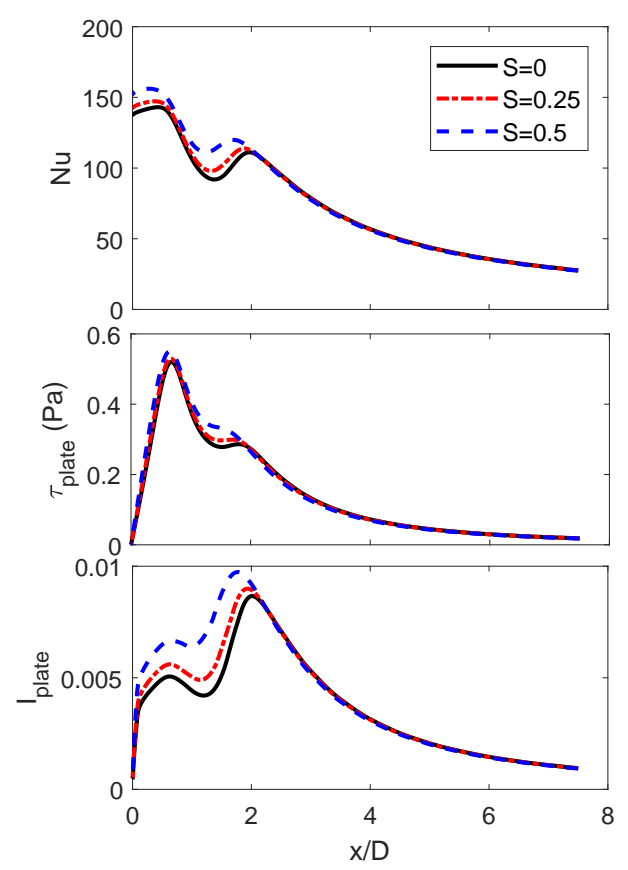

Figure 15: Plots of the friction factor on the Nusselt number (up), wall shear stress along the plate, $\tau_{\text {plate }}$ (centre) and turbulent intensity, $I_{\text {plate }}($ bottom), along the plate.

geometry and how is affected by the swirl. The axial velocity is highly influenced by the swirl, as shown in Figure 16. The increment in the axial velocity along the centre part of the jet is increased when the angular velocity of the pipe is augmented, as illustrated in Figure 17 for the fully-developed flow at the exit of the pipe. This effect is propagated from the pipe nozzle downstream up to the plate, where the flow impinges on it. The effect of swirl in the turbulent kinetic energy, $k$, for $H / D=2$ is shown in Figure 19, where the largest values of $k$ appear when $I$ is also large (see again Figure 15), since these two are related variables. In Figure 20, the turbulent kinetic energy is also compared with LES data from [88], showing similar contours.

Despite one can expect the increase in swirl intensity may result to a noticeable increase in the spread angle, this effect is not noticeable when using a rotating pipe mechanism with low-mid swirl intensity $(S=0-0.5)$. As a consequence, the radial velocity has been found to be essentially the same for $S=0, S=0.25$ and $S=0.5$, since it is driven by the impingement and, therefore, weakly affected by the increase in the swirl. This is also reflected in Figure 12, where vertical velocity profiles at different distances along the plate do not show large difference for the different $S$ values. In Figure 18 the effect of swirl in the azimuthal velocity at the $H / D=2$ is mostly observed along the nozzle lipline. A comparison with other mechanisms is discussed in the next section.

\subsection{Comparison of performance with other mechanisms}

As pointed out in Section 1 many different mechanisms may be used to introduce swirl to impinging jet flows, including pipes with spirals or vane-type swirl generators [11], swirl strips in pipes 


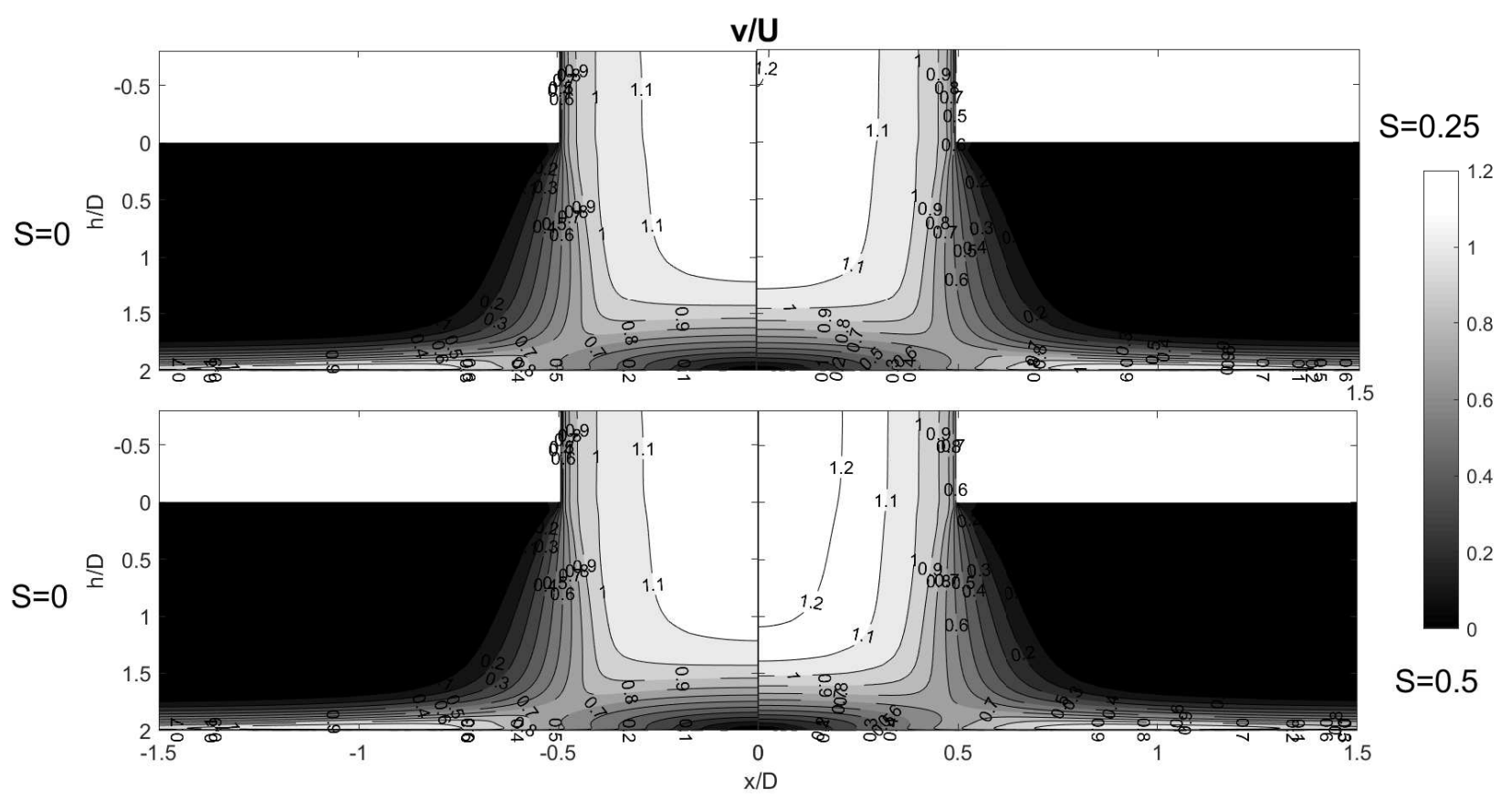

Figure 16: Effect of swirl on the velocity magnitude made dimensionless with the mean velocity $U$ at short nozzle-to-plate distance. $R e=23000$ and $H / D=2$.

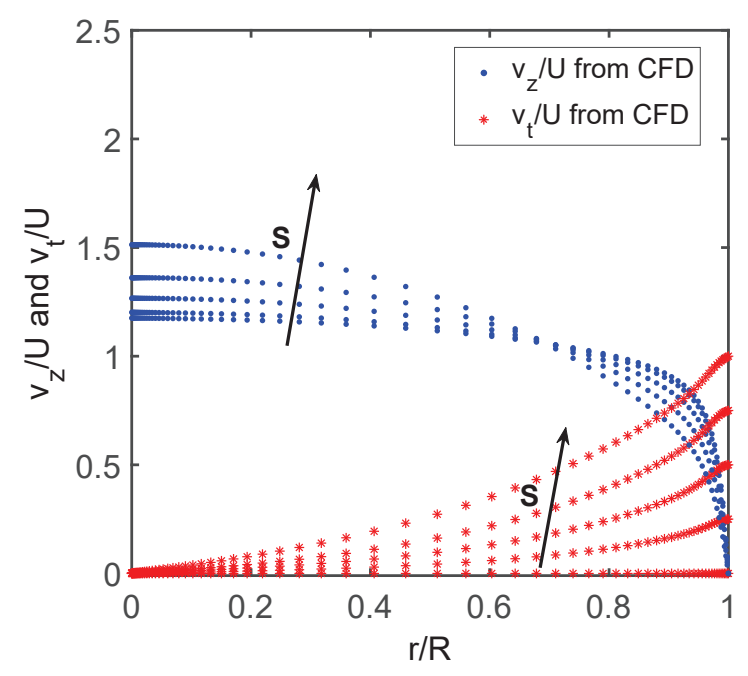

Figure 17: Effect of swirl on the axial and azimuthal velocity at the outflow section of the pipe (nozzle). The values of $\mathrm{S}$ for the plotted data are $0,0.25,0.5,0.75$ and 1.

[12], angled blades to force rotation [13, 14] or the use of tangential jets to induce the swirl [15]. In this paper the swirl is created by means of a rotating pipe. Depending on the swirl generator, the shape of the jet and the features that characterise it, can be completely different. Note that there are several definitions for the swirl number, which can be geometry-based [11, 12, 20] or fluid motion-based $[6,15]$. It is thus difficult to make a rigorous comparison even if all the test 


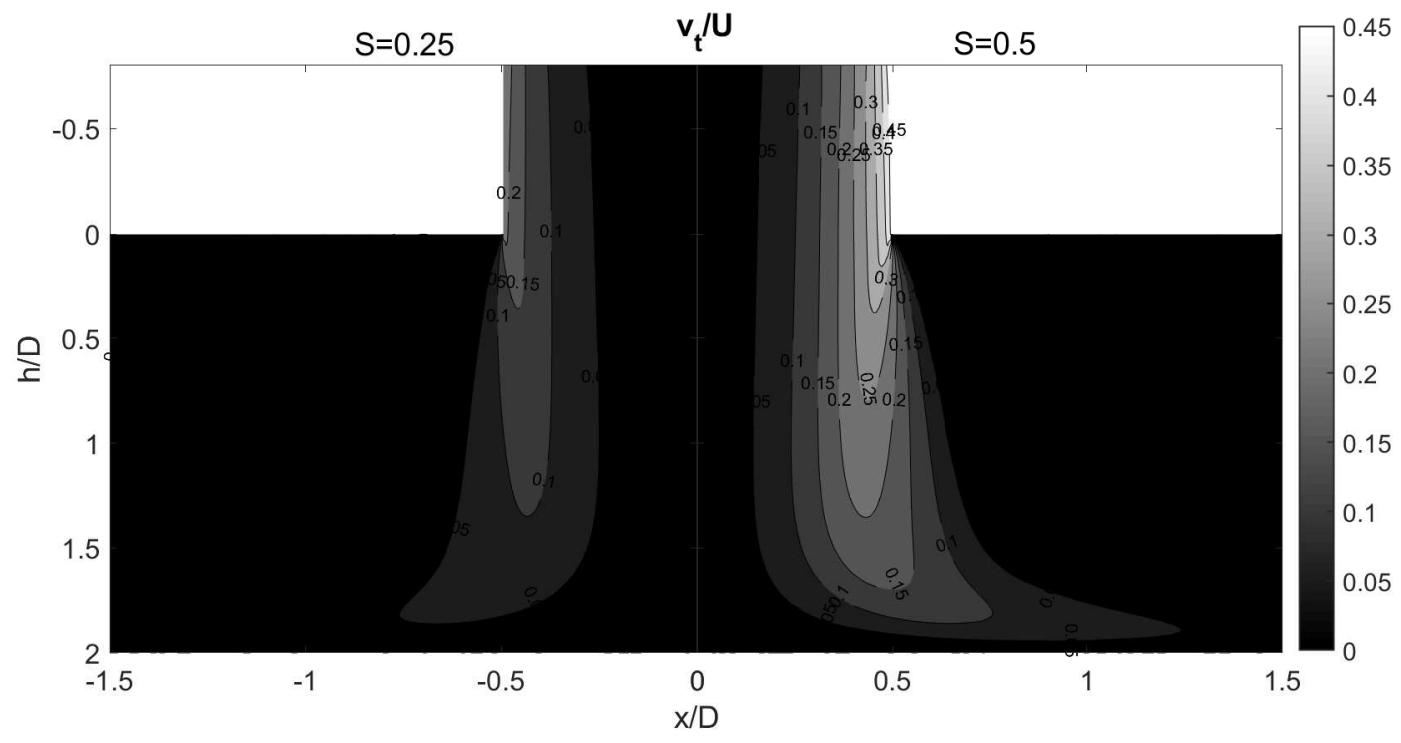

Figure 18: Effect of swirl on the azimuthal velocity made dimensionless with the mean velocity $U$ at short nozzle-to-plate distance. $R e=23000$ and $H / D=2$.

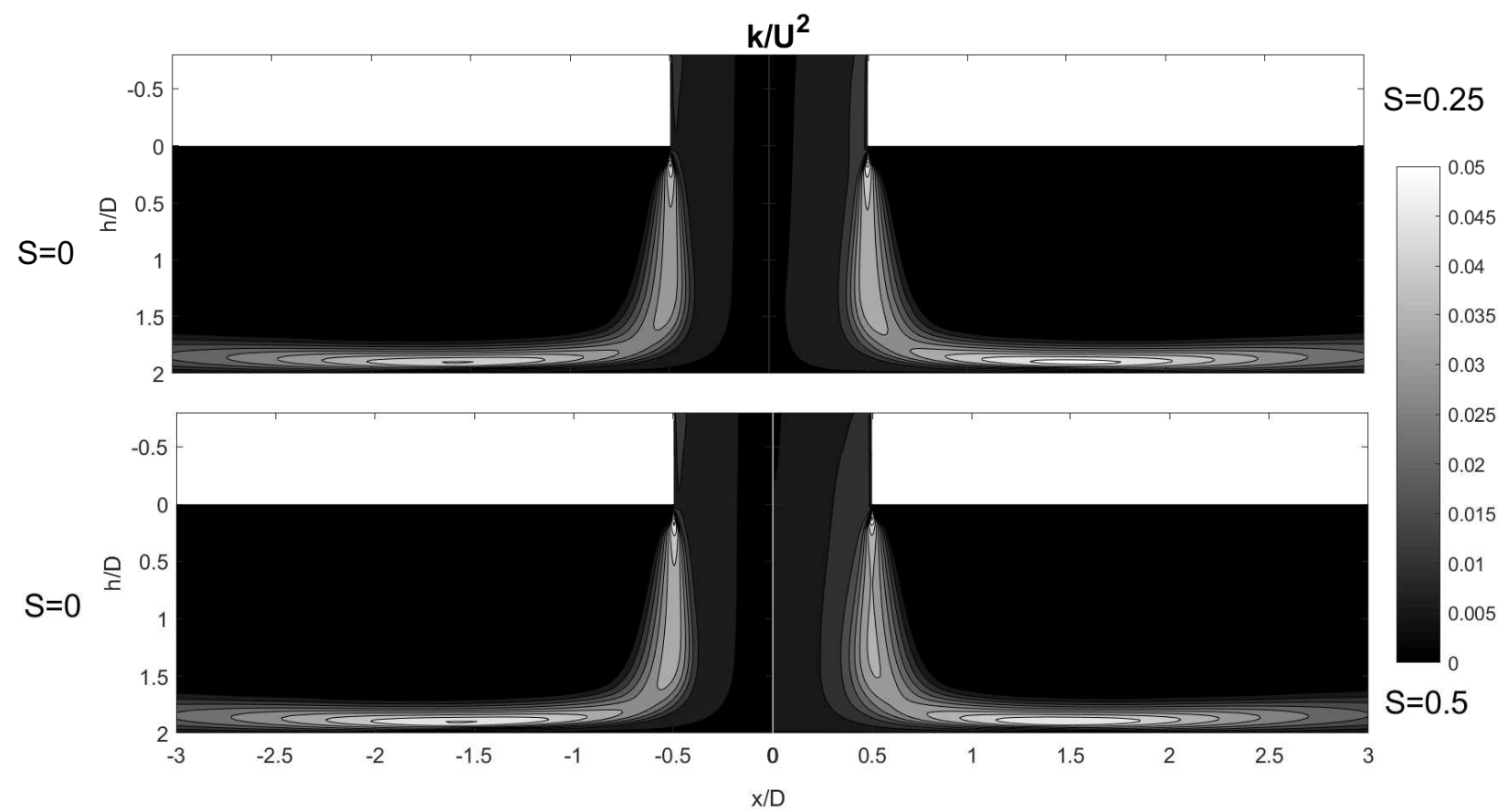

Figure 19: Effect of swirl on the turbulent kinetic energy made dimensionless with the mean velocity $U^{2}$ at short nozzle-to-plate distance. $R e=23000$ and $H / D=2$.

conditions (Re, $S, \operatorname{Pr}, H / D)$ are the same. However, it is relevant to show the performance of the mechanism in this paper versus the others for the sake of a qualitative reference. The radial distribution of the Nusselt number of the different mechanisms is plotted in Figure 21. A table 


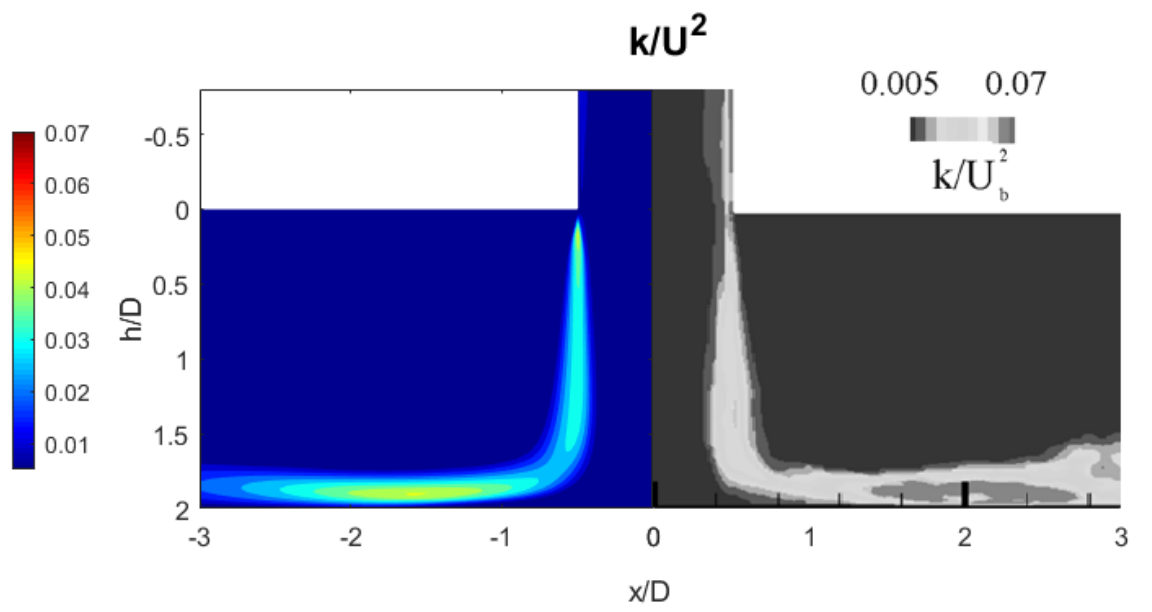

Figure 20: Comparison of the CFD turbulent kinetic energy contour plots (left) with LES from [88] (right). Images merged with permission of the correspondence author. $R e=23000, S=0$ and $H / D=2$.

with relevant information of the works considered for the comparison is shown in Table 2. All the cases with $S=0$ are referred to a round nozzle.

\begin{tabular}{c|c|c|c|c} 
& Re & $S$ & Mechanism & Type of work \\
\hline Lee et al. [1]] & 23000 & $\{0,0.21,0.44\}$ & spiral vane-type generators & Experimental \\
Baughn ES Shimizu [46] & 23700 & 0 & round pipe & Experimental \\
Behnia et al. [83] & 23000 & 0 & round pipe & CFD RANS \\
Yan E Saniei [15] & 23000 & $\{0,0.21,0.47\}$ & tangential jets at 120 degrees & Experimental \\
\hline
\end{tabular}

Table 2: Summary of the different papers considered for comparison.

At short distance $H / D=2$, the use of spiral vane-type generator as in Lee et al. produces a high peak in the radial Nusselt number, but at the highest $S$, the peak is decreased, as well as the value at the stagnation point, $N u_{0}$ (see Figure 21). With the use of tangential jets in Yan 8 Saniei, again a peak is generated, but less intense. The Nusselt number at the stagnation region is dramatically decreased when increasing $S$. For the rotating pipe the case scenario is the opposite: the Nusselt number is always increased with the increase of $S$. The $N u_{0}$ is also increased. This beneficial effect at short nozzle-to-plate distance has not been observed in any other mechanism in the literature. Depending on the mechanism, the jet generated and the way it spreads downstream shows different patterns. This spreading affects the impinging conditions which enhance the heat transfer due to the swirling jet turbulence. Typically, the shear layer growth and instabilities, entrainment of ambient air, and the jet decay are affected by the intensity of swirl [20, 90] and by other perturbations from the intrusiveness of the generation mechanisms. It has been reported by other authors that even the addition of light swirl can change significantly the behaviour of the jet [91]. Actually, the sudden expansion has been diminished by other researchers by the incorporation of a conical tip at the exit of the swirl generator, as in [20]. 


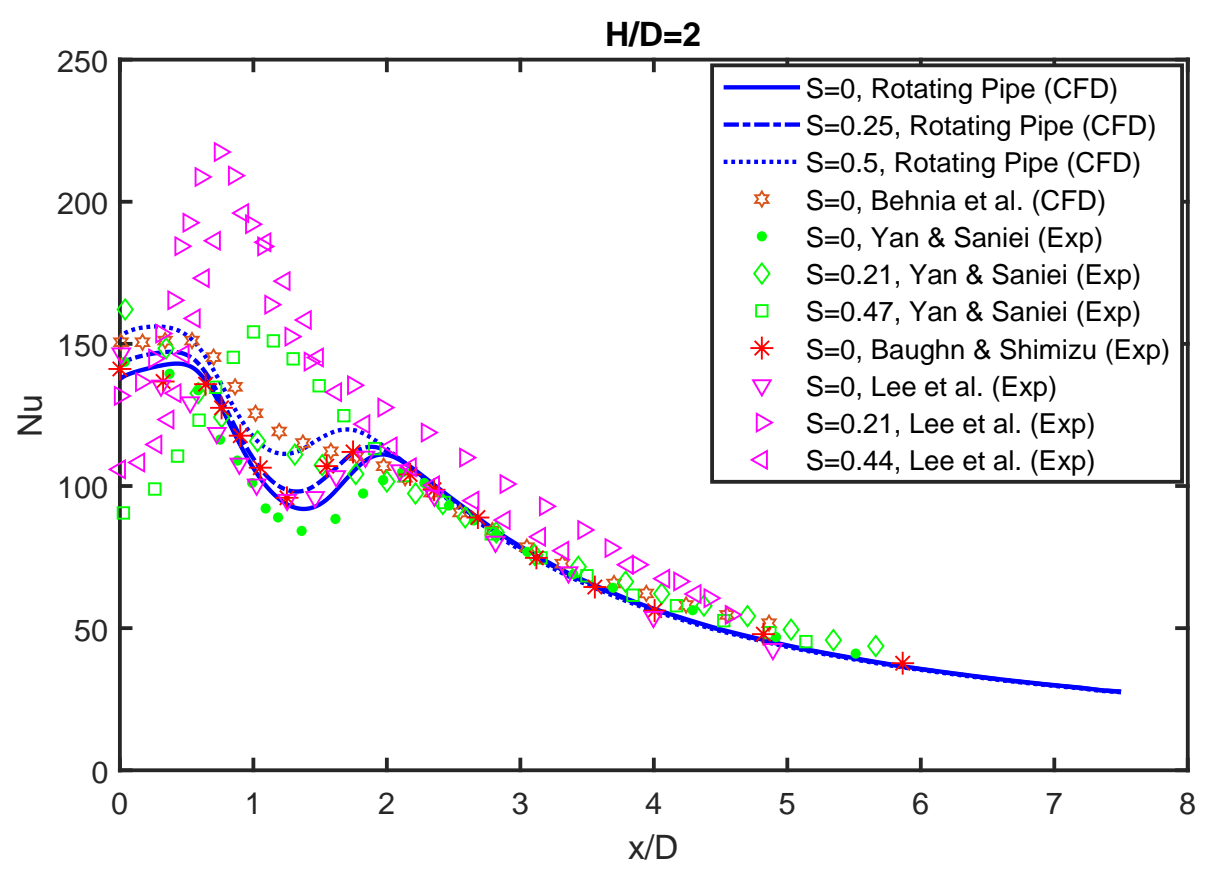

Figure 21: Comparison of the distribution of the radial Nusselt number and various swirl intensities with other existing data in the literature, with different swirl generation mechanisms for $H / D=2$. In the legends, Exp and $C F D$ stand for experimental and computational, respectively.

In Figure 22 and 23, it is shown the behaviour of $N u_{0}$ and $N u_{\text {avg }}$, respectively, at different nozzle-to-plate distances. Note that $N u_{a v g}$ is the average Nusselt number, which is evaluated only at the so-called impinging zone on the plate $0 \leq x / D \leq 2$ [92]. It can be seen that, for both $N u_{0}$ and $N u_{\text {avg }}$ at $H / D>6.5$, their evolution is reversed. It has been reported in the literature that for $H / D>10$ the effect of swirl may not be noticed in some applications [85], which is consistent with the numerical results here. The optimal value of $N u_{0}$ seems to be located in between $H / D=5.5$ and $H / D=6.5$ for all the swirl intensities considered in this investigation. This characteristic of the rotating pipe swirling jet may be relevant in applications whose interest is mainly the stagnation point at short distances. As shown, there is no great improvement of $N u_{0}$ by the addition of swirl up to $S=0.5$, since the same optimal value can be obtained without swirl, simply at a different $H / D$. However, other swirl intensities could provide greater heat transfer (either at stagnation point or globally), being of interest further research on this topic. The effect on $N u_{\text {avg }}$ has some resemblance with the effect observed for $N u_{0}$, as illustrated in Figure 23. It can be seen that, at least for the considered range of values for the parameters that govern the set-up, the optimal configuration for the highest $N u_{\text {avg }}$ takes place with $H / D \approx 5$ and $S=0.5$.

A study of correlations for $N u_{0}$ is carried out. These correlations are only built for the Nusselt number at the stagnation point because a simple correlation for the average Nusselt number (based on few constants) was not feasible. The suggested correlations for $N u_{0}$ are:

$$
\widehat{N u_{0}}=a(H / D)^{b}+c S+d, \text { for } 2 \leq H / D<6 \text { and } S \leq 0.5,
$$




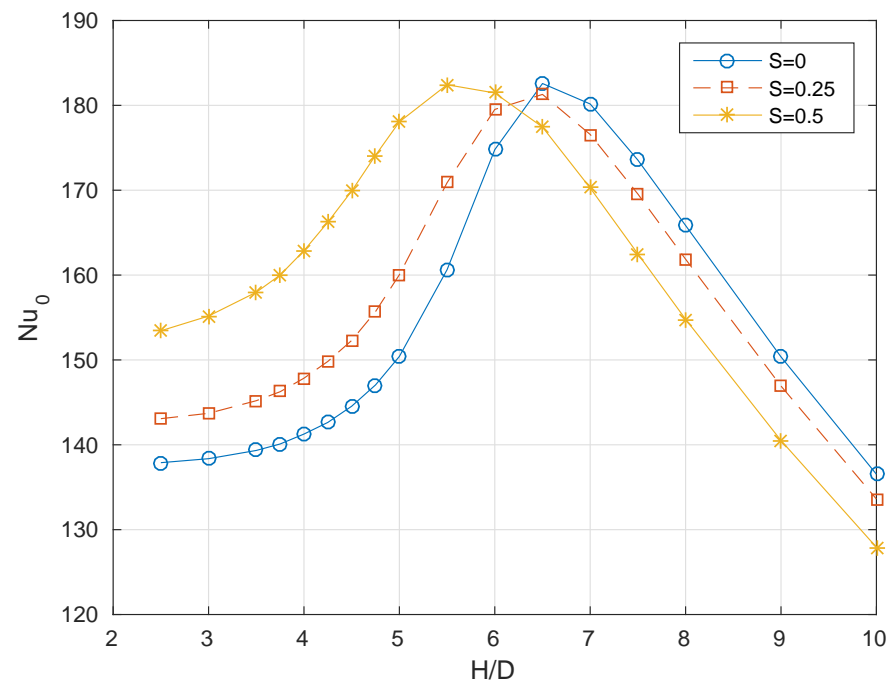

Figure 22: Evolution of the Nusselt number at the stagnation point, $N u_{0}$, with $R e=23000$, and different $S$ and $H / D$.

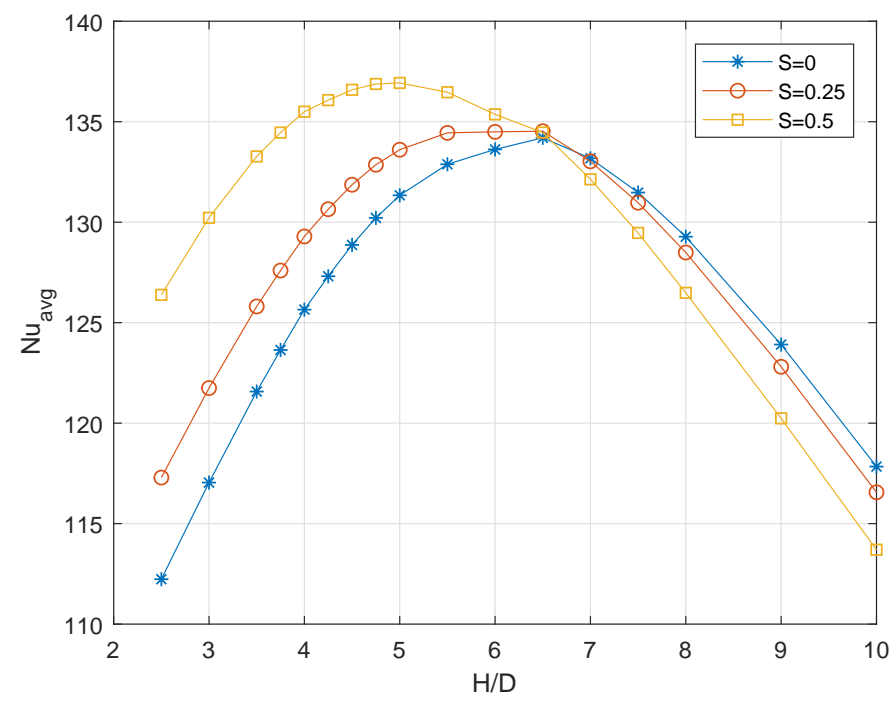

Figure 23: Evolution of the surface average Nusselt number, $N u_{\text {avg }}$, with $R e=23000$, and different $S$ and $H / D$.

$$
\widehat{N u_{0}}=a(H / D)+c S+d, \text { for } 6 \leq H / D \leq 10 \text { and } S \leq 0.5
$$

where $a, b, c$ and $d$ are constants for each correlation given in Table 3. The fitting error is calculated by using

$$
\text { error }=\frac{1}{N} \sum_{i=1}^{N} \frac{\left|N u_{0_{i}}-\widehat{N u_{0_{i}}}\right|}{N u_{0_{i}}} \times 100
$$


where $N u_{0_{i}}$ are the values from CFD (numerical values), $\widehat{N u_{0_{i}}}$ are the values predicted by the correlation (predicted values) and $N$ is the total number of data points. The accuracy of the approach is illustrated in Figure 24, with confidence intervals of a $\pm 3 \%$ of deviation.

\begin{tabular}{|c|c|c|c|c|c|}
\cline { 2 - 6 } \multicolumn{1}{c|}{} & $a$ & $b$ & $c$ & $d$ & error \\
\hline$H / D<6$ & 0.022 & 4.1968 & 43.593 & 132.5682 & $1.314 \%$ \\
\hline$H / D \geq 6$ & -14.0324 & - & -18.5310 & 277.5720 & $0.64 \%$ \\
\hline
\end{tabular}

Table 3: Constants for the correlations given in Eqs. (31) and (32).

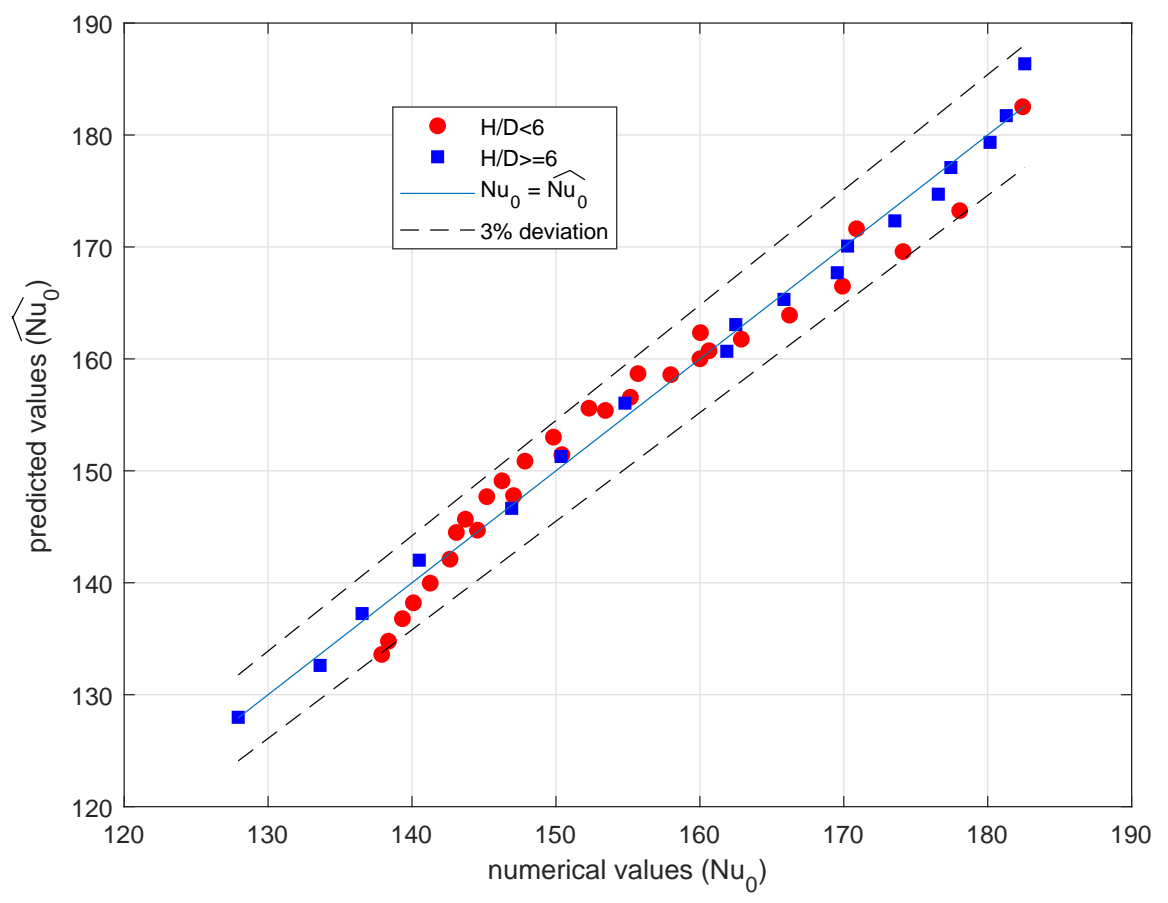

Figure 24: Numerical versus predicted values by the correlations for $N u_{0}$. The confidence intervals (dashed lines) are given by a $\pm 3 \%$ of deviation. The maximum and minimum values of $H / D$ in the plot are $H / D=2$ and $H / D=10$, respectively.

The conservation of the increase of $v_{z}$ at different $H / D$ distances, the growth of $v_{t}$ mostly along the nozzle lipline, and the weak influence on the spreading angle in the rotating pipe for the considered range of $S$ may be the reason to have better heat transfer at the stagnation region when increasing the swirl intensity at $H / D<6$. The jet flow at different swirl intensities is always impinging on a reduced stagnation region, being that location the most benefited from the properties added by the swirl. As a note, regardless of the differences in heat transfer by the different mechanisms, which might actually be small at large $H / D$ since the effect of swirl is less noticed [85], there are some advantages/disadvantages in the use of rotating pipe to consider.

1. With spiral vane-type generators, for each swirl intensity a new helical strip must be designed and built by Computer Numeric Control machining/3D printing. By using rotating pipes, 
only the angular velocity needs to be varied. The rotating pipe at the Fluid Dynamics Lab at University of Málaga is used for swirl break-down investigations because of the wide range of values of $S$ that can be achieved.

2. The intrusiveness of mechanisms such as blades $[93,13,14]$ oriented up to a zero degree for a non-swirling flow can still affect the jet. However, this can be avoided by dismantling the device, if possible.

3. Most mechanisms existing in industry and the literature, mentioned throughout the paper, generate flows with swirl that are highly Reynolds number dependent, i.e. $(S=S(R e))$. With rotating pipes, both $R e$ and $S$ can be controlled independently.

4. The disadvantages in the rotating pipe is the need of a pipe long enough to achieve a fullydeveloped state, and additionally, the need of a step-wise motor, which requires power supply.

To sum up, it is hard to fairly compare the different devices. First, because each mechanism generates a different jet flow pattern, and thus the resulting heat transfer distribution. Second, a mechanism can surpass others in terms of heat transfer, but there may be design or cost constrains. Other parameters can also be considered, such as the type of flow, variations on the surface of the plate [6], etc., which are out of the scope of this paper.

\section{Conclusions}

An extensive study of CFD simulations of a swirling impinging jet flow issuing from a rotating pipe has been carried out. A complete simulation has been done by splitting the large domain into two stages, in the spirit of versatility, in turbulence modelling and the use of periodic simulations in a pipe flow.

Simulation 1 concerns the simulation of the rotating pipe. The best performance was found with the Reynolds Stress turbulence Model (RSM), as eddy viscosity models tend to simulate the azimuthal velocity profile as solid rigid body rotation. This is because RSM takes into account the anisotropy in the generation of turbulence (directional effects of the Reynolds stress fields). Some RSM configurations do not properly mimic the experimental results and some are particularly problematic in solving the boundary layer. This is because the importance of the rotating wall in the contribution to swirl. The better the flow features of the boundary layer are solved, the more realistic the swirling flow. The best performance goes with Linear Pressure Strain and Enhanced Wall Function.

Simulation 2 concerns the simulation of heat transfer from a heated plate to an impinging jet. The Transition SST $k-\omega$ turbulence model with the Production Kato-Launder option performed the best. The Production Kato-Launder modification is very useful for impinging jets since it provides a correction to the common turbulent kinetic energy overproduction as a result of high values in the shear strain rate term in situations with sudden changes in velocity, as in impinging jets flows.

Numerical results are validated against a large variety of data in the literature, experimental and computational. When using rotating pipes with swirl intensity up to $S=0.5$, it is noticed that 
the spreading angle is minimally increased and the increase in azimuthal velocity is kept along the nozzle lipline. Moreover, there is an increase in the axial velocity in the central part of the axial jet. These features enhance the heat transfer at the stagnation region at short $H / D$ distances. However, further research showed that when $H / D$ is above $H / D=6.5$, the non-swirling flow shows better performance. It can be concluded that, for $S \leq 0.5$, the benefits of adding swirl are fading as $H / D$ increases. For the highest heat transfer at the impinging zone, the optimal configuration for the studied parameters is found to be for $S=0.5$ and $H / D \approx 5$. The advantages/disadvantages of rotating pipes in performance, cost and design have been also discussed.

To summarise, the separation of the full computational domain into two simulations using different turbulent models is shown to be an efficient and accurate approach. The numerical results presented in this paper aim to provide a valuable reference for industry and academia in the use of rotating pipes in order to generate swirling jets for heat transfer. It also aims to encourage further experimental and high-fidelity computational analyses to gain more knowledge on this heat transfer mechanism.

\section{Acknowledgments}

To be filled after peer review.

\section{References}

[1] RJK Wood, TF Jones, NJ Miles, and J Ganeshalingam. Upstream swirl-induction for reduction of erosion damage from slurries in pipeline bends. Wear, 250(1):770-778, 2001.

[2] Jos Derksen. Confined and agitated swirling flows with applications in chemical engineering. Flow, turbulence and combustion, 69(1):3-33, 2002.

[3] LIU Hengwei, L Zhonggliang, Feng Yongxun, G Keyu, and Y Tingmin. Characteristic of a supersonic swirling dehydration system of natural gas. Chinese Journal of Chemical Eng, $13(1): 9-12,2005$.

[4] BR Hollworth and M Durbin. Impingement cooling of electronics. Journal of heat transfer, 114(3):607-613, 1992.

[5] DH Lee, YS Chung, and PM Ligrani. Jet impingement cooling of chips equipped with multiple cylindrical pedestal fins. Journal of Electronic Packaging, 129(3):221-228, 2007.

[6] J Ortega-Casanova and FJ Granados-Ortiz. Numerical simulation of the heat transfer from a heated plate with surface variations to an impinging jet. International Journal of Heat and Mass Transfer, 76:128-143, 2014.

[7] Holger Martin. Heat and mass transfer between impinging gas jets and solid surfaces. In In: Advances in heat transfer. Volume 13. New York, Academic Press, Inc., 197r, p. 1-60., volume 13, pages 1-60, 1977. 
[8] R Viskanta. Heat transfer to impinging isothermal gas and flame jets. Experimental thermal and fluid science, 6(2):111-134, 1993.

[9] Thomas Hällqvist. Large eddy simulation of impinging jets with heat transfer. PhD thesis, Royal Institute of Technology, 2006.

[10] Naseem Uddin, Sven Olaf Neumann, and Bernhard Weigand. Les simulations of an impinging jet: On the origin of the second peak in the nusselt number distribution. International Journal of Heat and Mass Transfer, 57(1):356-368, 2013.

[11] Dae Hee Lee, Se Youl Won, Yun Taek Kim, and Young Suk Chung. Turbulent heat transfer from a flat surface to a swirling round impinging jet. International Journal of Heat and Mass Transfer, 45(1):223-227, 2002.

[12] Mao-Yu Wen and Kuen-Jang Jang. An impingement cooling on a flat surface by using circular jet with longitudinal swirling strips. International Journal of Heat and Mass Transfer, 46(24):4657-4667, 2003.

[13] J Ortega-Casanova. CFD and correlations of the heat transfer from a wall at constant temperature to an impinging swirling jet. International Journal of Heat and Mass Transfer, 55(21):5836-5845, 2012.

[14] Joaquín Ortega-Casanova. Numerical simulation of the heat transfer from a heated solid wall to an impinging swirling jet. INTECH Open Access Publisher, 2011.

[15] Xiaojun Yan and Nader Saniei. Heat transfer measurements from a flat plate to a swirling impinging jet. International Heat Transfer Conference August 23-28, Kyongiu, Korea, 5:497$502,1998$.

[16] Cecile Pera and Christian Angelberger. Large eddy simulation of a motored single-cylinder engine using system simulation to define boundary conditions: methodology and validation. SAE International Journal of Engines, 4(1):948-963, 2011.

[17] Federico Perini, Paul C Miles, and Rolf D Reitz. A comprehensive modeling study of incylinder fluid flows in a high-swirl, light-duty optical diesel engine. Computers \& Fluids, 105:113-124, 2014.

[18] Lionel Martinez, Adlène Benkenida, and Bénédicte Cuenot. A model for the injection boundary conditions in the context of $3 \mathrm{~d}$ simulation of diesel spray: methodology and validation. Fuel, 89(1):219-228, 2010.

[19] J.-H Li, Gary Page, and James Mcguirk. Turbulence modelling of the aerodynamic interaction of ogv wakes and diffuser flow. Hangkong Dongli Xuebao/Journal of Aerospace Power, 26:2302-2312, 102011.

[20] K Bakirci and K Bilen. Visualization of heat transfer for impinging swirl flow. Experimental thermal and fluid science, 32(1):182-191, 2007. 
[21] Zahir Uddin Ahmed. An experimental and numerical study of surface interactions in turbulent swirling jets. 2016.

[22] A White. Flow of a fluid in an axially rotating pipe. Journal of Mechanical Engineering Science, 6(1):47-52, 1964.

[23] Mitsukiyo Murakami and Kouji Kikuyama. Turbulent flow in axially rotating pipes. Journal of Fluids Engineering, 102(1):97-103, 1980.

[24] K Kikuyama, M Murakami, and K Nishibori. Development of three-dimensional turbulent boundary layer in an axially rotating pipe. Journal of Fluids Engineering, 105(2):154-160, 1983.

[25] Shigeki Imao, Motoyuki Itoh, and Takeyoshi Harada. Turbulent characteristics of the flow in an axially rotating pipe. International journal of heat and fluid flow, 17(5):444-451, 1996.

[26] Shuichiro Hirai, Toshimi Takagi, and Masaharu Matsumoto. Predictions of the laminarization phenomena in an axially rotating pipe flow. Journal of fluids engineering, 110(4):424-430, 1988.

[27] S Jakirlic, K Hanjalic, and C Tropea. Modeling rotating and swirling turbulent flows: a perpetual challenge. AIAA journal, 40(10):1984-1996, 2002.

[28] Toshio Kobayashi and Morio Yoda. Modified k-epsilon model for turbulent swirling flow in a straight pipe. JSME international journal, 30(259):66-71, 1987.

[29] Andrew Escue and Jie Cui. Comparison of turbulence models in simulating swirling pipe flows. Applied Mathematical Modelling, 34(10):2840-2849, 2010.

[30] MM Gibson and BA Younis. Calculation of swirling jets with a Reynolds Stress closure. Physics of Fluids (1958-1988), 29(1):38-48, 1986.

[31] AF Najafi, MH Saidi, MS Sadeghipour, and M Souhar. Boundary layer solution for the turbulent swirling decay flow through a fixed pipe: Sbr at the inlet. International journal of engineering science, 43(1):107-120, 2005.

[32] Yaser H Alahmadi and Andrzej F Nowakowski. Modified shear stress transport model with curvature correction for the prediction of swirling flow in a cyclone separator. Chemical Engineering Science, 147:150-165, 2016.

[33] CG Speziale, BA Younis, and SA Berger. Analysis and modelling of turbulent flow in an axially rotating pipe. Journal of Fluid Mechanics, 407:1-26, 2000.

[34] Stefan Wallin and Arne V Johansson. An explicit algebraic reynolds stress model for incompressible and compressible turbulent flows. Journal of Fluid Mechanics, 403:89-132, 2000.

[35] Svetlana V Poroseva, Stavros C Kassinos, Carlos A Langer, and William C Reynolds. Structure-based turbulence model: Application to a rotating pipe flow. Physics of Fluids, 14(4):1523-1532, 2002. 
[36] Koji KIKUYAMA, Mitsukiyo MURAKAMI, Kenji NISHIBORI, and Kazuhiko MAEDA. Flow in an axially rotating pipe: A calculation of flow in the saturated region. Bulletin of JSME, 26(214):506-513, 1983.

[37] MM Gibson and BE Launder. Ground effects on pressure fluctuations in the atmospheric boundary layer. Journal of Fluid Mechanics, 86(3):491-511, 1978.

[38] MR Malin and BA Younis. The prediction of turbulent transport in an axially rotating pipe. International communications in heat and mass transfer, 24(1):89-98, 1997.

[39] J Pruvost, J Legrand, and P Legentilhomme. Numerical investigation of bend and torus flows, Part I: effect of swirl motion on flow structure in U-bend. Chemical engineering science, 59(16):3345-3357, 2004.

[40] F Kaya and I Karagoz. Performance analysis of numerical schemes in highly swirling turbulent flows in cyclones. Current science, 94(10):1273-1278, 2008.

[41] F Nygård and HI Andersson. DNS of swirling turbulent pipe flow. International Journal for Numerical Methods in Fluids, 64(9):945-972, 2010.

[42] Luca Facciolo, Nils Tillmark, Alessandro Talamelli, and P Henrik Alfredsson. A study of swirling turbulent pipe and jet flows. Physics of Fluids, 19(3):035105, 2007.

[43] P Orlandi and M Fatica. Direct simulations of turbulent flow in a pipe rotating about its axis. Journal of Fluid Mechanics, 343:43-72, 1997.

[44] AA Feiz, M Ould-Rouis, and Guy Lauriat. Large Eddy Simulation of turbulent flow in a rotating pipe. International journal of heat and fluid flow, 24(3):412-420, 2003.

[45] J Eggels. Direct and Large Eddy Simulation of Turbulent Flow in a Cylindrical Pipe Geometry. PhD thesis, Delft University of Technology, The Netherlands, 1994.

[46] JW Baughn and S Shimizu. Heat transfer measurements from a surface with uniform heat flux and an impinging jet. Journal of Heat Transfer, 111(4):1096-1098, 1989.

[47] Zahir U Ahmed, Yasir M Al-Abdeli, and Miccal T Matthews. The effect of inflow conditions on the development of non-swirling versus swirling impinging turbulent jets. Computers $\&$ Fluids, 118:255-273, 2015.

[48] Puneet Gulati, Vadiraj Katti, and SV Prabhu. Influence of the shape of the nozzle on local heat transfer distribution between smooth flat surface and impinging air jet. International Journal of Thermal Sciences, 48(3):602-617, 2009.

[49] N Zuckerman and N Lior. Jet impingement heat transfer: physics, correlations, and numerical modeling. Advances in heat transfer, 39:565-631, 2006.

[50] B Sagot, G Antonini, A Christgen, and F Buron. Jet impingement heat transfer on a flat plate at a constant wall temperature. International Journal of Thermal Sciences, 47(12):1610-1619, 2008. 
[51] NiM Kerr and D Fraser. Swirl part 1: Effect on axisymmetrical turbulent jets. J. Inst. Fuel, 38(299):519, 1965.

[52] Frank M White and Isla Corfield. Viscous fluid flow, volume 3. McGraw-Hill New York, 2006.

[53] Glenn O Brown. The history of the darcy-weisbach equation for pipe flow resistance. Environmental and Water Resources History, 38(7):34-43, 2002.

[54] Fluent. Fluent 6.3 Users Guide. Fluent Incorporated, Centerra Resource Park, 10, Cavendish Court, Lebanon (NH) 03766 USA, 2005.

[55] F.-J. Granados-Ortiz, J. Ortega-Casanova, and C.-H. Lai. Uncertainty quantification and modelling of CFD simulations of a swirling turbulent jet created by a rotating pipe for application to heat transfer from a heated solid flat plate. In 1st ECCOMAS Thematic Conference on Uncertainty Quantification in Computational Sciences and Engineering. Crete Island, Greece, 2015.

[56] Yiqun Wang and Lifeng Yan. CFD studies on biomass thermochemical conversion. International journal of molecular sciences, 9(6):1108-1130, 2008.

[57] Brian Edward Launder and Dudley Brian Spalding. The numerical computation of turbulent flows. In Numerical prediction of flow, heat transfer, turbulence and combustion, pages 96-116. Elsevier, 1983.

[58] Chandra Laksham Vaidyaratna Jayatilleke. The influence of prandtl number and surface roughness on the resistance of the laminar sub-layer to momentum and heat transfer. 1966.

[59] S Kim. A near-wall treatment using wall functions sensitized to pressure gradient. Separated and complex flows, 1995.

[60] HC Chen and VC Patel. Near-wall turbulence models for complex flows including separation. AIAA journal, 26(6):641-648, 1988.

[61] T Jongen. Simulation and Modelling of Turbulent Incompressible Flows. PhD thesis, EPF Lausanne, 1992.

[62] BA Kader. Temperature and concentration profiles in fully turbulent boundary layers. International journal of heat and mass transfer, 24(9):1541-1544, 1981.

[63] FM White and GH Christoph. A simple new analysis of compressible turbulent twodimensional skin friction under arbitrary conditions. Technical report, RHODE ISLAND UNIV KINGSTON DEPT OF MECHANICAL ENGINEERING AND APPLIED MECHANICS, 1971.

[64] PG Huang, P Bradshaw, and TJ Coakley. Skin friction and velocity profile family for compressible turbulentboundary layers. AIAA journal, 31(9):1600-1604, 1993. 
[65] Sergio Galván, Marcelo Reggio, and Francois Guibault. Assessment study of k-epsilon turbulence models and near-wall modeling for steady state swirling flow analysis in draft tube using fluent. Engineering Applications of Computational Fluid Mechanics, 5(4):459-478, 2011.

[66] Najla El Gharbi, Rafik Absi, Ahmed Benzaoui, and EH Amara. Effect of near-wall treatments on airflow simulations. In International Conference on Computational Methods for Energy Engineering and Environment: ICCM3E. Sousse, Tunisia, 20-22 November, 2009, pages 185189, 2009.

[67] TJ Craft and BE Laundert. New wall-reflection model applied to the turbulent impinging jet. AIA A journal, 30(12):2970-2972, 1992.

[68] M Chmielewski and M Gieras. Three-zonal wall function for k- $\varepsilon$ turbulence models. Computational Methods in Science and Technology, 19(2):107-114, 2013.

[69] Y Yamada and S Imao. Swirling flow in an axially rotating pipe. Transactions of JSME, 46:1662-1670, 1980.

[70] Suhas V Patankar and D Brian Spalding. A calculation procedure for heat, mass and momentum transfer in three-dimensional parabolic flows. International Journal of Heat and Mass Transfer, 15(10):1787-1806, 1972.

[71] Ishmail B Celik, Urmila Ghia, Patrick J Roache, et al. Procedure for estimation and reporting of uncertainty due to discretization in CFD applications. Journal of fluids. EngineeringTransactions of the ASME, 130(7), 2008.

[72] J Panda and DK McLaughlin. Experiments on the instabilities of a swirling jet. Physics of Fluids, 6(1):263-276, 1994.

[73] Luca Facciolo. A study on axially rotating pipe and swirling jet flows. PhD thesis, KTH, 2006.

[74] BL Owsenek, T Cziesla, NK Mitra, and G Biswas. Numerical investigation of heat transfer in impinging axial and radial jets with superimposed swirl. International journal of heat and mass transfer, 40(1):141-147, 1996.

[75] SZ Shuja, BS Yilbas, and M Rashid. Confined swirling jet impingement onto an adiabatic wall. International journal of heat and mass transfer, 46(16):2947-2955, 2003.

[76] MA Herrada, C Del Pino, and J Ortega-Casanova. Confined swirling jet impingement on a flat plate at moderate reynolds numbers. Physics of Fluids, 21(1):013601, 2009.

[77] M Kato. The modelling of turbulent flow around stationary and vibrating square cylinders. Turbulent Shear Flow, 1:10-4, 1993.

[78] F Vittori, L Rojas-Solórzano, and M Pavageau. Comparative study of rans turbulence models for impinging jet simulations. In 9TH Engineer Annual Congress ASME USB, pages 23-25, 2011. 
[79] D Cooper, DC Jackson, Brian Edward Launder, and GX Liao. Impinging jet studies for turbulence model assessmentI. Flow-field experiments. International Journal of Heat and Mass Transfer, 36(10):2675-2684, 1993.

[80] Neil Zuckerman and Noam Lior. Radial slot jet impingement flow and heat transfer on a cylindrical target. Journal of Thermophysics and Heat Transfer, 21(3):548-561, 2007.

[81] Karel Petera and Martin Dostál. Heat transfer measurements and cfd simulations of an impinging jet. In EPJ Web of Conferences, volume 114, page 02091. EDP Sciences, 2016.

[82] Zahir U Ahmed, Yasir M Al-Abdeli, and Ferdinando G Guzzomi. Impingement pressure characteristics of swirling and non-swirling turbulent jets. Experimental Thermal and Fluid Science, 68:722-732, 2015.

[83] M Behnia, S Parneix, and Paul A Durbin. Prediction of heat transfer in an axisymmetric turbulent jet impinging on a flat plate. International Journal of Heat and Mass Transfer, 41(12):1845-1855, 1998.

[84] Robert Gardon and J Cahit Akfirat. The role of turbulence in determining the heat-transfer characteristics of impinging jets. International Journal of Heat and Mass Transfer, 8(10):12611272, 1965.

[85] Jungho Lee and Sang-Joon Lee. The effect of nozzle configuration on stagnation region heat transfer enhancement of axisymmetric jet impingement. International Journal of Heat and Mass Transfer, 43(18):3497-3509, 2000.

[86] Joseph Kestin, PF Maeder, and HE Wang. Influence of turbulence on the transfer of heat from plates with and without a pressure gradient. International Journal of Heat and Mass Transfer, 3(2):133-154, 1961.

[87] Pierre Grenson and Hugues Deniau. Large-eddy simulation of an impinging heated jet for a small nozzle-to-plate distance and high reynolds number. International Journal of Heat and Fluid Flow, 68:348-363, 2017.

[88] Naseem Uddin. Turbulence modeling of complex flows in CFD. PhD thesis, University of Sturrgart, 2008.

[89] Francis Shum-Kivan, Florent Duchaine, and Laurent Gicquel. Large-eddy simulation and conjugate heat transfer in a round impinging jet. In ASME Turbo Expo 2014: Turbine Technical Conference and Exposition, pages V05AT11A001-V05AT11A001. Citeseer, 2014.

[90] Bruce Douglas Pratte and JF Keffer. The swirling turbulent jet. Journal of Basic Engineering, 94(4):739-747, 1972.

[91] WG Rose. A swirling round turbulent jet: Part 1. mean-flow measurements. Journal of Applied Mechanics, 29(4):615-625, 1962. 
[92] Ajay Chatterjee. Heat transfer enhancement in laminar impinging flows with a non-newtonian inelastic fluid. Journal of Non-Newtonian Fluid Mechanics, 211:50-61, 2014.

[93] Joaquin Ortega-Casanova, Nicolas Campos, and Ramon Fernandez-Feria. Experimental study on sand bed excavation by impinging swirling jets. Journal of Hydraulic Research, 49(5):601610, 2011. 